\title{
Tracking chains revisited
}

\author{
Gunnar Wilken * \\ Structural Cellular Biology Unit \\ Okinawa Institute of Science and Technology \\ 1919-1 Tancha, Onna-son, 904-0495 Okinawa, Japan \\ wilken@oist.jp
}

October 17, 2018

\begin{abstract}
The structure $\mathcal{C}_{2}:=\left(1^{\infty}, \leq, \leq_{1}, \leq_{2}\right)$, introduced and first analyzed in [5], is shown to be elementary recursive. Here, $1^{\infty}$ denotes the proof-theoretic ordinal of the fragment $\Pi_{1}^{1}-\mathrm{CA}_{0}$ of second order number theory, or equivalently the set theory $\mathrm{KP} \ell_{0}$, which axiomatizes limits of models of Kripke-Platek set theory with infinity. The partial orderings $\leq_{1}$ and $\leq_{2}$ denote the relations of $\Sigma_{1}$ - and $\Sigma_{2}$-elementary substructure, respectively. In a subsequent article [1] we will show that the structure $\mathcal{C}_{2}$ comprises the core of the structure $\mathcal{R}_{2}$ of pure elementary patterns of resemblance of order 2. In [5] the stage has been set by showing that the least ordinal containing a cover of each pure pattern of order 2 is $1^{\infty}$. However, it is not obvious from 5 ] that $\mathcal{C}_{2}$ is an elementary recursive structure. This is shown here through a considerable disentanglement in the description of connectivity components of $\leq_{1}$ and $\leq_{2}$. The key to and starting point of our analysis is the apparatus of ordinal arithmetic developed in [7] and in Section 5 of [4, which was enhanced in [5], specifically for the analysis of $\mathcal{C}_{2}$.
\end{abstract}

\section{Introduction}

Let $\mathcal{R}_{2}=\left(\mathrm{Ord} ; \leq, \leq_{1}, \leq_{2}\right)$ be the structure of ordinals with standard linear ordering $\leq$ and partial orderings $\leq_{1}$ and $\leq_{2}$, simultaneously defined by induction on $\beta$ in

$$
\alpha \leq_{i} \beta: \Leftrightarrow\left(\alpha ; \leq, \leq_{1}, \leq_{2}\right) \preceq_{\Sigma_{i}}\left(\beta ; \leq, \leq_{1}, \leq_{2}\right)
$$

where $\preceq_{\Sigma_{i}}$ is the usual notion of $\Sigma_{i}$-elementary substructure (without bounded quantification), see [1, 2] for fundamentals and groundwork on elementary patterns of resemblance. Pure patterns of order 2 are the finite isomorphism types of $\mathcal{R}_{2}$. The core of $\mathcal{R}_{2}$ consists of the union of isominimal realizations of these patterns within $\mathcal{R}_{2}$, where a finite substructure of $\mathcal{R}_{2}$ is called isominimal, if it is pointwise minimal (with respect to increasing enumerations) among all substructures of $\mathcal{R}_{2}$ isomorphic to it, and where an isominimal substructure of $\mathcal{R}_{2}$ realizes a pattern $P$, if it is isomorphic to $P$. It is a basic observation, cf. [2], that the class of pure patterns of order 2 is contained in the class $\mathcal{R F}_{2}$ of respecting forests of order 2: finite structures $P$ over the language $\left(\leq_{0}, \leq_{1}, \leq_{2}\right)$ where $\leq_{0}$ is a linear ordering and $\leq_{1}, \leq_{2}$ are forests such that $\leq_{2} \subseteq_{1} \leq_{1} \subseteq \leq_{0}$ and $\leq_{i+1}$ respects $\leq_{i}$, i.e. $p \leq_{i} q \leq_{i} r \& p \leq_{i+1} r$ implies $p \leq_{i+1} q$ for all $p, q, r \in P$, for $i=0,1$.

In [5] we showed that every pattern has a cover below $1^{\infty}$, the least such ordinal. Here, an order isomorphism (embedding) is a cover (covering, respectively) if it maintains the relations $\leq_{1}$ and $\leq_{2}$. The ordinal of $\mathrm{KP} \ell_{0}$ is therefore least such that there exist arbitrarily long finite $\leq_{2}$-chains. Moreover, by determination of enumeration functions of (relativized) connectivity components of $\leq_{1}$ and $\leq_{2}$ we were able to describe these relations in terms of classical ordinal notations. The central observation in connection with this is that every ordinal below $1^{\infty}$ is the greatest element in a $\leq_{1}$-chain in which $\leq_{1}$ - and $\leq_{2}$-chains alternate. We called such chains tracking chains as they provide all $\leq_{2}$-predecessors and the greatest $\leq_{1}$-predecessors insofar as they exist.

\footnotetext{
*This article is a pre-print of a chapter in Sets and Computations, Lecture Notes Series Vol. 33, Institute for Mathematical Sciences, National University of Singapore, C World Scientific Publishing Company (2017), see [10]. The author would like to acknowledge the Institute for Mathematical Sciences of the National University of Singapore for its partial support of this work during the "Interactions" week of the workshop Sets and Computations in April 2015.
} 
In the present article we will review and slightly extend the ordinal arithmetical toolkit and then verify through a disentangling reformulation, that [5] in fact yields an elementary recursive characterization of the restriction of $\mathcal{R}_{2}$ to the structure $\mathcal{C}_{2}=\left(1^{\infty} ; \leq, \leq_{1}, \leq_{2}\right)$. It is not obvious from [5] that $\mathcal{C}_{2}$ is an elementary recursive structure since several proofs there make use of transfinite induction up to $1^{\infty}$, which allowed for a somewhat shorter argumentation there. We will summarize the results in [5] to a sufficient and convenient degree. As a byproduct, [5] will become considerably more accessible. We will prove the equivalence of the arithmetical descriptions of $\mathcal{C}_{2}$ given in [5] and here. Note that the equivalence of this elementary recursive characterization with the original structure based on elementary substructurehood is proven in Section 7 of [5], using full transfinite induction up to the ordinal of $\mathrm{KP} \ell_{0}$. In this article we rely on this result and henceforth identify $\mathcal{C}_{2}$ with its arithmetical characterization given in [5] and further illuminated in Section 5 of the present article, where we also show that the finite isomorphism types of the arithmetical $\mathcal{C}_{2}$ are respecting forests of order 2 , without relying on semantical characterization of the arithmetical $\mathcal{C}_{2}$.

With these preparations out of the way we will be able to provide, in a subsequent article [11], an algorithm that assigns an isominimal realization within $\mathcal{C}_{2}$ to each respecting forest of order 2 , thereby showing that each such respecting forest is in fact (up to isomorphism) a pure pattern of order 2. The approach is to formulate the corresponding theorem flexibly so that isominimal realizations above certain relativizing tracking chains are considered. There we will also define an elementary recursive function that assigns descriptive patterns $P(\alpha)$ to ordinals $\alpha \in 1^{\infty}$. A descriptive pattern for an ordinal $\alpha$ in the above sense is a pattern, the isominimal realization of which contains $\alpha$. Descriptive patterns will be given in a way that makes a canonical choice for normal forms, since in contrast to the situation in $\mathcal{R}_{1}^{+}$, cf. [9, 4, there is no unique notion of normal form in $\mathcal{R}_{2}$. The chosen normal forms will be of least possible cardinality.

The mutual order isomorphisms between hull and pattern notations that will be given in [1] enable classification of a new independence result for $\mathrm{KP} \ell_{0}$ : We will demonstrate that the result by Carlson in [3, according to which the collection of respecting forests of order 2 is well-quasi ordered with respect to coverings, cannot be proven in $\mathrm{KP} \ell_{0}$ or, equivalently, in the restriction $\Pi_{1}^{1}-\mathrm{CA}_{0}$ of second order number theory to $\Pi_{1}^{1}$-comprehension and set induction. On the other hand, we know that transfinite induction up to the ordinal $1^{\infty}$ of $\mathrm{KP} \ell_{0}$ suffices to show that every pattern is covered [5].

This article therefore delivers the first part of an in depth treatment of the insights and results presented in a lecture during the "Interactions" week of the workshop Sets and Computations, held at the Institute for Mathematical Sciences of the National University of Singapore in April 2015.

\section{Preliminaries}

The reader is assumed to be familiar with basics of ordinal arithmetic (see e.g. [6]) and the ordinal arithmetical tools developed in [7] and Section 5 of [4]. See the index at the end of [7] for quick access to its terminology. Section 2 of [9] (2.1-2.3) provides a summary of results from [7]. As mentioned before, we will build upon [5], the central concepts and results of which will be reviewed here and in the next section. For detailed reference see also the index of [5].

\section{$2.1 \quad$ Basics}

Here we recall terminology already used in [5] (Section 2) for the reader's convenience. Let $\mathbb{P}$ denote the class of additive principal numbers, i.e. nonzero ordinals that are closed under ordinal addition, that is the image of ordinal exponentiation to base $\omega$. Let $\mathbb{L}$ denote the class of limits of additive principal numbers, i.e. the limit points of $\mathbb{P}$, and let $\mathbb{M}$ denote the class of multiplicative principal numbers, i.e. nonzero ordinals closed under ordinal multiplication. By $\mathbb{E}$ we denote the class of epsilon numbers, i.e. the fixed-points of $\omega$-exponentiation.

We write $\alpha={ }_{\mathrm{ANF}} \alpha_{1}+\ldots+\alpha_{n}$ if $\alpha_{1}, \ldots, \alpha_{n} \in \mathbb{P}$ such that $\alpha_{1} \geq \ldots \geq \alpha_{n}$, which is called the representation of $\alpha$ in additive normal form, and $\alpha==_{\mathrm{NF}} \beta+\gamma$ if the expansion of $\beta$ into its additive normal form (ANF) in the sum $\beta+\gamma$ syntactically results in the additive normal form of $\alpha$. The Cantor normal form representation of an ordinal $\alpha$ is given by $\alpha={ }_{\mathrm{CNF}} \omega^{\alpha_{1}}+\ldots+\omega^{\alpha_{n}}$ where $\alpha_{1} \geq \ldots \geq \alpha_{n}$ with $\alpha>\alpha_{1}$ unless $\alpha \in \mathbb{E}$. For $\alpha=_{\mathrm{ANF}} \alpha_{1}+\ldots+\alpha_{n}$, we define $\operatorname{mc}(\alpha):=\alpha_{1}$ and end $(\alpha):=\alpha_{n}$. We set end(0):=0. Given ordinals $\alpha, \beta$ with $\alpha \leq \beta$ we write $-\alpha+\beta$ for the unique $\gamma$ such that $\alpha+\gamma=\beta$. As usual let $\alpha-\beta$ be 0 if $\beta \geq \alpha, \gamma$ if $\beta<\alpha$ and there exists the minimal $\gamma$ s.t. $\alpha=\gamma+\beta$, and $\alpha$ otherwise.

For $\alpha \in$ Ord we denote the least multiplicative principal number greater than $\alpha$ by $\alpha^{\mathbb{M}}$. Notice that if $\alpha \in \mathbb{P}$, $\alpha>1$, say $\alpha=\omega^{\alpha^{\prime}}$, we have $\alpha^{\mathbb{M}}=\alpha^{\omega}=\omega^{\alpha^{\prime} \cdot \omega}$. For $\alpha \in \mathbb{P}$ we use the following notations for multiplicative normal 
form:

1. $\alpha==_{\mathrm{NF}} \eta \cdot \xi$ if and only if $\xi=\omega^{\xi_{0}} \in \mathbb{M}$ (i.e. $\xi_{0} \in\{0\} \cup \mathbb{P}$ ) and either $\eta=1$ or $\eta=\omega^{\eta_{1}+\ldots+\eta_{n}}$ such that $\eta_{1}+\ldots+\eta_{n}+\xi_{0}$ is in additive normal form. When ambiguity is unlikely, we sometimes allow $\xi$ to be of a form $\omega^{\xi_{1}+\ldots+\xi_{m}}$ such that $\eta_{1}+\ldots+\eta_{n}+\xi_{1}+\ldots+\xi_{m}$ is in additive normal form.

2. $\alpha={ }_{\mathrm{MNF}} \alpha_{1} \cdot \ldots \alpha_{k}$ if and only if $\alpha_{1}, \ldots, \alpha_{k}$ is the unique decreasing sequence of multiplicative principal numbers, the product of which is equal to $\alpha$.

For $\alpha \in \mathbb{P}, \alpha={ }_{\mathrm{MNF}} \alpha_{1} \cdot \ldots \cdot \alpha_{k}$, we write $\operatorname{mf}(\alpha)$ for $\alpha_{1}$ and lf $(\alpha)$ for $\alpha_{k}$. Note that if $\alpha \in \mathbb{P}-\mathbb{M}$ then $\operatorname{lf}(\alpha) \in \mathbb{M}^{>1}$ and $\alpha={ }_{\mathrm{NF}} \bar{\alpha} \cdot \operatorname{lf}(\alpha)$ where the definition of $\bar{\alpha}$ given in [7] for limits of additive principal numbers is extended to ordinals $\alpha$ of a form $\alpha=\omega^{\alpha^{\prime}+1}$ by $\bar{\alpha}:=\omega^{\alpha^{\prime}}$, see Section 5 of $[4$.

Given $\alpha, \beta \in \mathbb{P}$ with $\alpha \leq \beta$ we write $(1 / \alpha) \cdot \beta$ for the uniquely determined ordinal $\gamma \leq \beta$ such that $\alpha \cdot \gamma=\beta$. Note that with the representations $\alpha=\omega^{\alpha^{\prime}}$ and $\beta=\omega^{\beta^{\prime}}$ we have

$$
(1 / \alpha) \cdot \beta=\omega^{-\alpha^{\prime}+\beta^{\prime}} .
$$

For any $\alpha$ of a form $\omega^{\alpha^{\prime}}$ we write $\log (\alpha)$ for $\alpha^{\prime}$, and we set $\log (0):=0$. For an arbitrary ordinal $\beta$ we write $\operatorname{logend}(\beta)$ for $\log (\operatorname{end}(\beta))$.

\subsection{Relativized notation systems $\mathrm{T}^{\tau}$}

Settings of relativization are given by ordinals from $\mathbb{E}_{1}:=\{1\} \cup \mathbb{E}$ and frequently indicated by Greek letters, preferably $\sigma$ or $\tau$. Clearly, in this context $\tau=1$ denotes the trivial setting of relativization. For a setting $\tau$ of relativization we define $\tau^{\infty}:=\mathrm{T}^{\tau} \cap \Omega_{1}$ where $\mathrm{T}^{\tau}$ is defined as in [7] and reviewed in Section 2.2 of [9]. $\mathrm{T}^{\tau}$ is the closure of parameters below $\tau$ under addition and the stepwise, injective, and fixed-point free collapsing functions $\vartheta_{k}$ the domain of which is $\mathrm{T}^{\tau} \cap \Omega_{k+2}$, where $\vartheta^{\tau}:=\vartheta_{0}$ is relativized to $\tau$. As in [5], most considerations will be confined to the segment $1^{\infty}$. Translation between different settings of relativization, see Section 6 of [7], is effective on the term syntax and enjoys convenient invariance properties regarding the operators described below, as was verified in [7, 4], and [5. We therefore omit the purely technical details here.

\subsection{Refined localization}

The notion of $\tau$-localization (Definition 2.11 of [9]) and its refinement to $\tau$-fine-localization by iteration of the operator ${ }^{-}$, see Definitions 5.1 and 5.5 of [4, continue to be essential as they locate ordinals in terms of closure properties (fixed-point level and limit point thinning). These notions are effectively derived from the term syntax. We refer to Subsection 2.3 of [9] and Section 5 of [4] for a complete picture of these concepts. In the present article, the operator ${ }^{-}$is mostly used to decompose ordinals that are not multiplicative principal, i.e. if $\alpha={ }_{\mathrm{NF}} \eta \cdot \xi$ where $\eta>1$ and $\xi \in \mathbb{M}$, then $\bar{\alpha}=\eta$. The notion of $\tau$-localization enhanced with multiplicative decomposition turns out to be the appropriate tool for the purposes of the present article, whereas general $\tau$-fine-localization will re-enter the picture through the notion of closedness in a subsequent article [11].

\subsection{Operators related to connectivity components}

The function $\log$ (logend) is described in $\mathrm{T}^{\tau}$-notation in Lemma 2.13 of $[9]$, and for $\beta=\vartheta^{\tau}(\eta) \in \mathrm{T}^{\tau}$ where $\eta<\Omega_{1}$ we have

$$
\log ((1 / \tau) \cdot \beta)= \begin{cases}\eta+1 & \text { if } \eta=\varepsilon+k \text { where } \varepsilon \in \mathbb{E}^{>\tau}, k<\omega \\ \eta & \text { otherwise. }\end{cases}
$$

The foregoing distinction reflects the property of $\vartheta$-functions to omit fixed points.

The operators $\iota_{\tau, \alpha}$ indicating the fixed-point level, $\zeta_{\alpha}^{\tau}$ displaying the degree of limit point thinning, and their combination $\lambda_{\alpha}^{\tau}$ measuring closure properties of ordinals $\alpha \in \mathrm{T}^{\tau}$ are as in Definitions 2.14 and 2.18 of [9], which also reviews the notion of base transformation $\pi_{\sigma, \tau}$ and its smooth interaction with these operators.

The operator $\lambda_{\alpha}^{\tau}$ already played a central role in the analysis of $\mathcal{R}_{1}^{+}$-patterns as it displays the number of $\leq_{1}$-connectivity components that are $\leq_{1}$-connected to the component with index $\alpha$ in a setting of relativization $\tau \in \mathbb{E}_{1}$, cf. Lemma 2.31 part (a) of [9]. It turns out that $\lambda_{\alpha}^{\tau}$ plays a similar role in $\mathcal{R}_{2}$, see below.

In order to avoid excessive repetition of formal definitions from [5] we continue to describe operators and functions introduced for analysis of $\mathcal{C}_{2}$ in [5] in terms of their meaning in the context of $\mathcal{C}_{2}$. Those (relativized) 
$\leq_{1}$-components, the enumeration index of which is an epsilon number, give rise to infinite $\leq_{1}$-chains, along which new $\leq_{2}$-components arise. Omitting from these $\leq_{1}$-chains those elements that have a $\leq_{2}$-predecessor in the chain and enumerating the remaining elements, we obtain the so-called $\nu$-functions, see Definition 4.4 of [5], and the $\mu$-operator provides the length of such enumerations up to the final newly arising $\leq_{2}$-component, cf. the remark before Definition 4.4 of [5]. This terminal point on a main line, at which the largest newly arising $\leq_{2}$-component originates, is crucial for understanding the structure $\mathcal{C}_{2}$. Note that in general the terminal point has an infinite increasing continuation in the $\leq_{1}$-chain under consideration, leading to $\leq_{2}$-components which have isomorphic copies below, i.e. which are not new. Recall Convention 2.9 of [9].

Definition 2.1 (3.4 of [5]) Let $\tau \in \mathbb{E}_{1}$ and $\alpha \in\left(\tau, \tau^{\infty}\right) \cap \mathbb{E}$, say $\alpha=\vartheta^{\tau}(\Delta+\eta)$ where $\eta<\Omega_{1}$ and $\Delta=\Omega_{1} \cdot(\lambda+k)$ such that $\lambda \in\{0\} \cup \operatorname{Lim}$ and $k<\omega$. We define

$$
\mu_{\alpha}^{\tau}:=\omega^{\iota_{\tau, \alpha}(\lambda)+\chi^{\alpha}\left(\iota_{\tau, \alpha}(\lambda)\right)+k} .
$$

The $\chi$-indicator occurring above is given in Definition 3.1 of [5] and indicates whether the maximum $\leq_{2}$-component starting from an ordinal on the infinite $\leq_{1}$-chain under consideration itself $\leq_{1}$-reconnects to that chain which we called a main line. The question remains which $\leq_{1}$-component starting from such a point on a main line is the largest that is also $\leq_{2}$-connected to it. This is answered by the $\varrho$-operator:

Definition 2.2 (3.9 of [5]) Let $\alpha \in \mathbb{E}, \beta<\alpha^{\infty}$, and $\lambda \in\{0\} \cup \operatorname{Lim}, k<\omega$ be such that $\operatorname{logend}(\beta)=\lambda+k$. We define

$$
\varrho_{\beta}^{\alpha}:=\alpha \cdot\left(\lambda+k \dot{-} \chi^{\alpha}(\lambda)\right)
$$

Now, the terminal point on a main line, given as, say, $\nu_{\mu_{\alpha}^{\tau}}^{\boldsymbol{\tau}, \alpha}$ with a setting of relativization $\boldsymbol{\tau}=\left(\tau_{1}, \ldots, \tau_{n}\right)$ that will be discussed later and $\tau=\tau_{n}, \alpha \in \mathbb{E}^{>\tau}$, connects to $\lambda_{\alpha}^{\tau}$-many $\leq_{1}$-components. The following lemma is a direct consequence of the respective definitions.

Lemma 2.3 (3.12 of [5]) Let $\tau \in \mathbb{E}_{1}$ and $\alpha=\vartheta^{\tau}(\Delta+\eta) \in\left(\tau, \tau^{\infty}\right) \cap \mathbb{E}$. Then we have

1. $\iota_{\tau, \alpha}(\Delta)=\varrho_{\mu_{\alpha}^{\tau}}^{\alpha}$ and hence $\lambda_{\alpha}^{\tau}=\varrho_{\mu_{\alpha}^{\tau}}^{\alpha}+\zeta_{\alpha}^{\tau}$.

2. $\varrho_{\beta}^{\alpha} \leq \lambda_{\alpha}^{\tau}$ for every $\beta \leq \mu_{\alpha}^{\tau}$. For $\beta<\mu_{\alpha}^{\tau}$ such that $11 \chi^{\alpha}(\beta)=0$ we even have $\varrho_{\beta}^{\alpha}+\alpha \leq \lambda_{\alpha}^{\tau}$.

3. If $\mu_{\alpha}^{\tau}<\alpha$ we have $\mu_{\alpha}^{\tau}<\alpha \leq \lambda_{\alpha}^{\tau}<\alpha^{2}$, while otherwise

$$
\max \left(\left(\mu_{\alpha}^{\tau}+1\right) \cap \mathbb{E}\right)=\max \left(\left(\lambda_{\alpha}^{\tau}+1\right) \cap \mathbb{E}\right) .
$$

4. If $\lambda_{\alpha}^{\tau} \in \mathbb{E}^{>\alpha}$ we have $\mu_{\alpha}^{\tau}=\lambda_{\alpha}^{\tau} \cdot \omega$ in case of $\chi^{\alpha}\left(\lambda_{\alpha}^{\tau}\right)=1$ and $\mu_{\alpha}^{\tau}=\lambda_{\alpha}^{\tau}$ otherwise.

Notice that we have $\mu_{\alpha}^{\tau}=\iota_{\tau, \alpha}(\Delta)=\operatorname{mc}\left(\lambda_{\alpha}^{\tau}\right)$ whenever $\mu_{\alpha}^{\tau} \in \mathbb{E}^{>\alpha}$.

\section{Tracking sequences and their evaluation}

\subsection{Maximal and minimal $\mu$-coverings}

The following sets of sequences are crucial for the description of settings of relativization, which in turn is the key to understanding the structure of connectivity components in $\mathcal{C}_{2}$.

Definition 3.1 (4.2 of [5]) Let $\tau \in \mathbb{E}_{1}$. A nonempty sequence $\left(\alpha_{1}, \ldots, \alpha_{n}\right)$ of ordinals in the interval $\left[\tau, \tau^{\infty}\right)$ is called a $\tau$-tracking sequence if

1. $\left(\alpha_{1}, \ldots, \alpha_{n-1}\right)$ is either empty or a strictly increasing sequence of epsilon numbers greater than $\tau$.

2. $\alpha_{n} \in \mathbb{P}, \alpha_{n}>1$ if $n>1$.

3. $\alpha_{i+1} \leq \mu_{\alpha_{i}}^{\tau}$ for every $i \in\{1, \ldots, n-1\}$.

By $\mathrm{TS}^{\tau}$ we denote the set of all $\tau$-tracking sequences. Instead of $\mathrm{TS}^{1}$ we also write $\mathrm{TS}$.

\footnotetext{
${ }^{1}$ This condition is missing in [5]. However, that inequality was only applied under this condition, cf. Def. 5.1 and L. 5.7 of $[5]$.
} 
According to Lemma 3.5 of [5] the length of a tracking sequence is bounded in terms of the largest index of $\vartheta$-functions in the term representation of the first element of the sequence.

Definition 3.2 Let $\tau \in \mathbb{E}_{1}, \alpha \in \mathbb{E} \cap\left(\tau, \tau^{\infty}\right)$, and $\beta \in \mathbb{P} \cap\left(\alpha, \alpha^{\infty}\right)$. A sequence $\left(\alpha_{0}, \ldots, \alpha_{n+1}\right)$ where $\alpha_{0}=\alpha$, $\alpha_{n+1}=\beta,\left(\alpha_{1}, \ldots, \alpha_{n+1}\right) \in \mathrm{TS}^{\alpha}$, and $\alpha<\alpha_{1} \leq \mu_{\alpha}^{\tau}$ is called a $\mu$-covering from $\alpha$ to $\beta$.

Lemma 3.3 Any $\mu$-covering from $\alpha$ to $\beta$ is a subsequence of the $\alpha$-localization of $\beta$.

Proof. Let $\left(\alpha_{0}, \ldots, \alpha_{n+1}\right)$ be a $\mu$-covering from $\alpha$ to $\beta$. Stepping down from $\alpha_{n+1}$ to $\alpha_{0}$, Lemmas 3.5 of [5] and $4.9,6.5$ of [7] apply to show that the $\alpha$-localization of $\beta$ is the successive concatenation of the $\alpha_{i}$-localization of $\alpha_{i+1}$ for $i=1, \ldots, n$, modulo translation between the respective settings of relativization.

Definition 3.4 Let $\tau \in \mathbb{E}_{1}$.

1. For $\alpha \in \mathbb{P} \cap\left(\tau, \tau^{\infty}\right)$ we define $\max -\operatorname{cov}^{\tau}(\alpha)$ to be the longest subsequence $\left(\alpha_{1}, \ldots, \alpha_{n+1}\right)$ of the $\tau$-localization of $\alpha$ which satisfies $\tau<\alpha_{1}, \alpha_{n+1}=\alpha$, and which is $\mu$-covered, i.e. which satisfies $\alpha_{i+1} \leq \mu_{\alpha_{i}}^{\tau}$ for $i=1, \ldots, n$.

2. For $\alpha \in \mathbb{E} \cap\left(\tau, \tau^{\infty}\right)$ and $\beta \in \mathbb{P} \cap\left(\alpha, \alpha^{\infty}\right)$ we denote the shortest subsequence $\left(\beta_{0}, \beta_{1}, \ldots, \beta_{n+1}\right)$ of the $\alpha$-localization of $\beta$ which is a $\mu$-covering from $\alpha$ to $\beta$ by $\min -\operatorname{cov}^{\alpha}(\beta)$, if such sequence exists.

We recall the notion of the tracking sequence of an ordinal, for greater clarity only for multiplicative principals at this stage.

Definition 3.5 (cf. 3.13 of [5]) Let $\tau \in \mathbb{E}_{1}$ and $\alpha \in \mathbb{M} \cap\left(\tau, \tau^{\infty}\right)$ with $\tau$-localization $\tau=\alpha_{0}, \ldots, \alpha_{n}=\alpha$. The tracking sequence of $\alpha$ above $\tau, \operatorname{ts}^{\tau}(\alpha)$, is defined as follows. If there exists the largest index $i \in\{1, \ldots, n-1\}$ such that $\alpha \leq \mu_{\alpha_{i}}^{\tau}$, then

$$
\operatorname{ts}^{\tau}(\alpha):=\operatorname{ts}^{\tau}\left(\alpha_{i}\right) \frown(\alpha)
$$

otherwise $\operatorname{ts}^{\tau}(\alpha):=(\alpha)$.

Definition 3.6 Let $\tau \in \mathbb{E}_{1}, \alpha \in \mathbb{E} \cap\left(\tau, \tau^{\infty}\right), \beta \in \mathbb{P} \cap\left(\alpha, \alpha^{\infty}\right)$, and let $\alpha=\alpha_{0}, \ldots, \alpha_{n+1}=\beta$ be the $\alpha$-localization of $\beta$. If there exists the least index $i \in\{0, \ldots, n\}$ such that $\alpha_{i}<\beta \leq \mu_{\alpha_{i}}^{\tau}$, then

$$
\operatorname{mts}^{\alpha}(\beta):=\operatorname{mts}^{\alpha}\left(\alpha_{i}\right) \frown(\beta),
$$

otherwise $\operatorname{mts}^{\alpha}(\beta):=(\alpha)$.

Note that $\operatorname{mts}^{\alpha}(\beta)$ reaches $\beta$ if and only if it is a $\mu$-covering from $\alpha$ to $\beta$.

Lemma 3.7 Fix $\tau \in \mathbb{E}_{1}$.

1. For $\alpha \in \mathbb{P} \cap\left(\tau, \tau^{\infty}\right)$ let $\max -\operatorname{cov}^{\tau}(\alpha)=\left(\alpha_{1}, \ldots, \alpha_{n+1}\right)=\boldsymbol{\alpha}$. If $\alpha_{1}<\alpha$ then $\boldsymbol{\alpha}$ is a $\mu$-covering from $\alpha_{1}$ to $\alpha$ and $\operatorname{mts}^{\alpha_{1}}(\alpha) \subseteq \boldsymbol{\alpha}$.

2. If $\alpha \in \mathbb{M} \cap\left(\tau, \tau^{\infty}\right)$ then $\max -\operatorname{cov}^{\tau}(\alpha)=\operatorname{ts}^{\tau}(\alpha)$.

3. Let $\alpha \in \mathbb{E} \cap\left(\tau, \tau^{\infty}\right)$ and $\beta \in \mathbb{P} \cap\left(\alpha, \alpha^{\infty}\right)$. Then $\min -\operatorname{cov}^{\alpha}(\alpha)$ exists if and only if $\operatorname{mts}^{\alpha}(\beta)$ is a $\mu$-covering from $\alpha$ to $\beta$, in which case these sequences are equal, characterizing the lexicographically maximal $\mu$-covering from $\alpha$ to $\beta$.

Proof. These are immediate consequences of the definitions.

Recall Definition 3.16 from [5], which for $\tau \in \mathbb{E}_{1}, \alpha \in \mathbb{E} \cap\left(\tau, \tau^{\infty}\right)$ defines $\widehat{\alpha}$ to be the minimal $\gamma \in \mathbb{M}^{>\alpha}$ such that $\operatorname{ts}^{\alpha}(\gamma)=(\gamma)$ and $\mu_{\alpha}^{\tau}<\gamma$.

Lemma 3.8 Let $\tau \in \mathbb{E}_{1}, \alpha \in \mathbb{E} \cap\left(\tau, \tau^{\infty}\right)$, and $\beta \in \mathbb{M} \cap\left(\alpha, \alpha^{\infty}\right)$. Then $\operatorname{mts}^{\alpha}(\beta)$ is a $\mu$-covering from $\alpha$ to $\beta$ if and only if $\beta<\widehat{\alpha}$. This holds if and only if for $\operatorname{ts}^{\alpha}(\beta)=\left(\beta_{1}, \ldots, \beta_{m}\right)$ we have $\beta_{1} \leq \mu_{\alpha}^{\tau}$. 
Proof. Suppose first that $\operatorname{mts}^{\alpha}(\beta)$ is a $\mu$-covering from $\alpha$ to $\beta$. Then $\alpha$ is an element of the $\tau$-localization of $\beta$, and modulo term translation we obtain the $\tau$-localization of $\beta$ by concatenating the $\tau$-localization of $\alpha$ with the $\alpha$-localization of $\beta$. By Lemma 3.7 we therefore have $\operatorname{mts}^{\alpha}(\beta) \subseteq(\alpha) \frown \operatorname{ts}^{\alpha}(\beta)$ where $\beta_{1} \leq \mu_{\alpha}$. Let $\gamma \in \mathbb{M} \cap(\alpha, \beta]$ be given. Then by Lemma 3.15 of [5] we have

$$
\left(\gamma_{1}, \ldots, \gamma_{k}\right):=\operatorname{ts}^{\alpha}(\gamma) \leq_{\operatorname{lex}} \operatorname{ts}^{\alpha}(\beta)
$$

so $\gamma_{1} \leq \beta_{1} \leq \mu_{\alpha}$, and hence $\beta<\widehat{\alpha}$.

Toward proving the converse, suppose that $\beta<\widehat{\alpha}$. We have $\operatorname{ts}^{\alpha}\left(\beta_{1}\right)=\left(\beta_{1}\right)$, so $\beta_{1} \leq \mu_{\alpha}$ since $\beta_{1}<\widehat{\alpha}$. This implies that $\operatorname{mts}^{\alpha}(\beta)$ reaches $\beta$ as a subsequence of $(\alpha) \frown \operatorname{ts}^{\alpha}(\beta)$.

Definition 3.9 (4.3 of [5]) Let $\tau \in \mathbb{E}_{1}$. A sequence $\boldsymbol{\alpha}$ of ordinals below $\tau^{\infty}$ is a $\tau$-reference sequence if

1. $\boldsymbol{\alpha}=()$ or

2. $\boldsymbol{\alpha}=\left(\alpha_{1}, \ldots, \alpha_{n}\right) \in \mathrm{TS}^{\tau}$ such that $\alpha_{n} \in \mathbb{E}^{>\alpha_{n-1}}$ (where $\left.\alpha_{0}:=\tau\right)$.

We denote the set of $\tau$-reference sequences by $\mathrm{RS}^{\tau}$. In case of $\tau=1$ we simply write $\mathrm{RS}$ and call its elements reference sequences.

Definition 3.10 (c.f. 4.9 of [5]) For $\gamma \in \mathbb{M} \cap 1^{\infty}$ and $\varepsilon \in \mathbb{E} \cap 1^{\infty}$ let $\operatorname{sk}_{\gamma}(\varepsilon)$ be the maximal sequence $\delta_{1}, \ldots, \delta_{l}$ such that (setting $\delta_{0}:=1$ )

1. $\delta_{1}=\varepsilon$ and

2. if $i \in\{1, \ldots, l-1\} \& \delta_{i} \in \mathbb{E}^{>\delta_{i-1}} \& \gamma \leq \mu_{\delta_{i}}$, then $\delta_{i+1}=\overline{\mu_{\delta_{i}} \cdot \gamma}$.

$\operatorname{Remark}([\mathbf{5}])$. Lemma 3.5 of [5] guarantees that the above definition terminates. We have $\left(\delta_{1}, \ldots, \delta_{l-1}\right) \in \mathrm{RS}$ and $\left(\delta_{1}, \ldots, \delta_{l}\right) \in$ TS. Notice that $\gamma \leq \delta_{i}$ for $i=2, \ldots, l$.

Definition 3.11 Let $\alpha \frown \beta \in \operatorname{RS}$ and $\gamma \in \mathbb{M}$.

1. If $\gamma \in(\beta, \widehat{\beta})$, let $\operatorname{mts}^{\beta}(\gamma)=\boldsymbol{\eta} \frown(\varepsilon, \gamma)$ and define

$$
\mathrm{h}_{\gamma}(\boldsymbol{\alpha} \frown \beta):=\boldsymbol{\alpha}^{\frown} \boldsymbol{\eta}^{\frown} \operatorname{sk}_{\gamma}(\varepsilon) .
$$

2. If $\gamma \in(1, \beta]$ and $\gamma \leq \mu_{\beta}$ then

$$
\mathrm{h}_{\gamma}(\boldsymbol{\alpha} \frown \beta):=\boldsymbol{\alpha}^{\frown} \operatorname{sk}_{\gamma}(\beta) .
$$

3. If $\gamma \in(1, \beta]$ and $\gamma>\mu_{\beta}$ then

$$
\mathrm{h}_{\gamma}\left(\boldsymbol{\alpha}^{\frown \beta)}:=\boldsymbol{\alpha}^{\frown \beta} .\right.
$$

Remark. In 1. let ts ${ }^{\beta}(\gamma)=:\left(\gamma_{1}, \ldots, \gamma_{m}\right)$. Then we have $\gamma_{1} \leq \mu_{\beta}$ according to Lemma 3.17 of [5], so that $\operatorname{mts}^{\beta}(\gamma)$ reaches $\gamma, \beta \leq \varepsilon<\gamma \leq \mu_{\varepsilon}$ and $\beta<\mu_{\beta}$. In 2. we have $\operatorname{sk}_{\gamma}(\beta)=\left(\beta, \overline{\mu_{\beta} \cdot \gamma}\right)$ with $\gamma \leq \overline{\mu_{\beta} \cdot \gamma} \leq \beta$ in case of $\mu_{\beta} \leq \beta$. In 3. we have $\mu_{\beta}<\gamma \leq \beta$ and hence $\operatorname{sk}_{\gamma}(\beta)=(\beta)$.

Lemma 3.12 Let $\boldsymbol{\alpha} \frown \beta \in \mathrm{RS}$ and $\gamma \in \mathbb{M}$. Then $\mathrm{h}_{\gamma}(\boldsymbol{\alpha} \frown \beta)$ is of a form $\boldsymbol{\alpha} \frown \boldsymbol{\eta} \frown \operatorname{sk}_{\gamma}(\varepsilon)$ where $\boldsymbol{\eta}=\left(\eta_{1}, \ldots, \eta_{r}\right)$, $r \geq 0, \eta_{1}=\beta, \eta_{r+1}:=\varepsilon$, and $\operatorname{sk}_{\gamma}(\varepsilon)=\left(\delta_{1}, \ldots, \delta_{l+1}\right), l \geq 0$, with $\delta_{1}=\varepsilon$. We have

$$
\operatorname{lf}\left(\delta_{l+1}\right) \geq \gamma,
$$

and for $\boldsymbol{\tau}^{\frown} \sigma \in \mathrm{TS}$, where $\boldsymbol{\tau}=\left(\tau_{1}, \ldots, \tau_{s}\right)$ and $\tau_{s+1}:=\sigma$, such that $\boldsymbol{\alpha} \frown \beta \subseteq \boldsymbol{\tau}^{\frown \sigma \text { and }} \mathrm{h}_{\gamma}(\boldsymbol{\alpha} \frown \beta)<_{\operatorname{lex}} \boldsymbol{\tau}^{\frown \sigma \text { we }}$ either have

1. $\boldsymbol{\tau}=\boldsymbol{\alpha}^{\frown} \boldsymbol{\eta} \frown \boldsymbol{\delta}_{\mid i}$ for some $i \in\{1, \ldots, l+1\}$ and $\sigma=_{\mathrm{NF}} \delta_{i+1} \cdot \sigma^{\prime}$ for some $\sigma^{\prime}<\gamma$, setting $\delta_{l+2}:=1$, or

2. $\boldsymbol{\tau}_{\uparrow_{s_{0}}}=\boldsymbol{\alpha} \frown \boldsymbol{\eta}_{\uparrow_{r_{0}}}$ for some $r_{0} \in[1, r]$ and $s_{0} \leq s$ such that $\eta_{r_{0}+1}<\tau_{s_{0}+1}$, in which case we have $\mu_{\tau_{j}}<\gamma$ for all $j \in\left\{s_{0}+1, \ldots, s\right\}$, and $\mu_{\sigma}<\gamma$ if $\sigma \in \mathbb{E}^{>\tau_{s}}$. 
Proof. Suppose first that $\boldsymbol{\tau}$ is a maximal initial segment $\boldsymbol{\alpha}^{\frown} \boldsymbol{\eta} \frown \boldsymbol{\delta}_{\uparrow_{i}}$ for some $i \in\{1, \ldots, l+1\}$.

In the case $i=l+1$ we have $\delta_{l+1} \in \mathbb{E}^{>\delta_{l}}$ and $\mu_{\delta_{l+1}}<\gamma \leq \delta_{l+1}$, so $\sigma \leq \mu_{\delta_{l+1}}<\gamma$, and we also observe that $\boldsymbol{\tau}^{\frown} \sigma$ could not be extended further. Now suppose that $i \leq l$. Then $\delta_{i+1}<\sigma \leq \mu_{\delta_{i}}$, and since $\gamma \leq \delta_{i+1}=\overline{\mu_{\delta_{i}} \cdot \gamma} \leq \mu_{\delta_{i}}$, we obtain $\sigma=\delta_{i+1} \cdot \sigma^{\prime}$ for some $\sigma^{\prime} \in(1, \gamma)$.

Otherwise $\boldsymbol{\tau}$ must be of the form given in part 2 of the claim. This implies $\gamma \in \mathbb{M} \cap(\beta, \widehat{\beta})$ and $\operatorname{mts}^{\beta}(\gamma)=$ $\boldsymbol{\eta}^{-}(\varepsilon, \gamma)$. Let us assume, toward contradiction, there existed a least $j \in\left\{s_{0}+1, \ldots, s+1\right\}$ such that $\tau_{j} \in \mathbb{E}^{>\tau_{j-1}}$ and $\gamma \leq \mu_{\tau_{j}}$. Then $\boldsymbol{\eta}_{r_{r_{0}}}-\left(\tau_{s_{0}+1}, \ldots, \tau_{j}, \gamma\right)$ is a $\mu$-covering from $\beta$ to $\gamma$, hence by part 3 of Lemma 3.7 it must be



Corollary 3.13 For $\boldsymbol{\alpha} \frown \beta \in \mathbb{R S}$ and $\gamma, \delta \in \mathbb{M}$ such that $\delta \in(1, \gamma)$ we have

$$
\mathrm{h}_{\gamma}(\boldsymbol{\alpha} \frown \beta) \leq_{\operatorname{lex}} \mathrm{h}_{\delta}(\boldsymbol{\alpha} \frown \beta) .
$$

\subsection{Evaluation}

Definition 3.14 Let $\boldsymbol{\alpha} \sim \beta \in \mathrm{TS}$, where $\boldsymbol{\alpha}=\left(\alpha_{1}, \ldots, \alpha_{n}\right), n \geq 0, \beta==_{\mathrm{MNF}} \beta_{1} \ldots \cdot \beta_{k}$, and set $\alpha_{0}:=1, \alpha_{n+1}:=\beta$, $h:=\mathrm{ht}_{1}\left(\alpha_{1}\right)+1$, and $\gamma_{i}:=\operatorname{ts}^{\alpha_{i-1}}\left(\alpha_{i}\right), i=1, \ldots, n$,

$$
\gamma_{n+1}:= \begin{cases}(\beta) & \text { if } \beta \leq \alpha_{n} \\ \operatorname{ts}^{\alpha_{n}}\left(\beta_{1}\right)-\beta_{2} & \text { if } k>1, \beta_{1} \in \mathbb{E}^{>\alpha_{n}} \& \beta_{2} \leq \mu_{\beta_{1}} \\ \operatorname{ts}^{\alpha_{n}}\left(\beta_{1}\right) & \text { otherwise, }\end{cases}
$$

and write $\gamma_{i}=\left(\gamma_{i, 1}, \ldots, \gamma_{i, m_{i}}\right), i=1, \ldots, n+1$. Then define

$$
\operatorname{lSeq}(\boldsymbol{\alpha} \frown \beta):=\left(m_{1}, \ldots, m_{n+1}\right) \in[h]^{\leq h} .
$$

Let $\beta^{\prime}:=1$ if $k=1$ and $\beta^{\prime}:=\beta_{2} \cdot \ldots \cdot \beta_{k}$ otherwise. We define $\mathrm{o}(\boldsymbol{\alpha} \sim \beta)$ recursively in $\operatorname{lSeq}(\boldsymbol{\alpha} \sim \beta)$, as well as auxiliary parameters $n_{0}(\boldsymbol{\alpha} \frown \beta)$ and $\gamma(\boldsymbol{\alpha} \frown \beta)$, which are set to 0 where not defined explicitly.

1. $\mathrm{o}((1)):=1$.

2. If $\beta_{1} \leq \alpha_{n}$, then $\mathrm{o}(\boldsymbol{\alpha} \frown \beta):=_{\mathrm{NF}} \mathrm{o}(\boldsymbol{\alpha}) \cdot \beta$.

3. If $\beta_{1} \in \mathbb{E}^{>\alpha_{n}}, k>1$, and $\beta_{2} \leq \mu_{\beta_{1}}$, then set $n_{0}(\boldsymbol{\alpha} \frown \beta):=n+1, \gamma(\boldsymbol{\alpha} \frown \beta):=\beta_{1}$, and define

$$
\mathrm{o}(\boldsymbol{\alpha} \frown \beta):={ }_{\mathrm{NF}} \mathrm{o}\left(\mathrm{h}_{\beta_{2}}\left(\boldsymbol{\alpha} \frown \beta_{1}\right)\right) \cdot \beta^{\prime} .
$$

4. Otherwise. Then setting

$$
n_{0}:=n_{0}(\boldsymbol{\alpha} \frown \beta):=\max \left(\left\{i \in\{1, \ldots, n+1\} \mid m_{i}>1\right\} \cup\{0\}\right),
$$

define

$$
\mathrm{o}(\boldsymbol{\alpha} \frown \beta):=_{\mathrm{NF}} \begin{cases}\beta & \text { if } n_{0}=0 \\ \mathrm{o}\left(\mathrm{h}_{\beta_{1}}\left(\boldsymbol{\alpha}_{\left.\right|_{n_{0}-1}} \frown \gamma\right)\right) \cdot \beta & \text { if } n_{0}>0,\end{cases}
$$

where $\gamma:=\gamma(\boldsymbol{\alpha} \frown \beta):=\gamma_{n_{0}, m_{n_{0}}-1}$.

Remark. As indicated in writing $=_{\mathrm{NF}}$ in the above definition, we obtain terms in multiplicative normal form denoting the values of o. The fixed points of o, i.e. those $\boldsymbol{\alpha} \neg \beta$ that satisfy o $(\boldsymbol{\alpha} \neg \beta)=\beta$ are therefore characterized by 1 . and 4 . for $n_{0}=0$.

Recall Definition 3.13 of [5], extending Definition 3.5] to additive principal numbers that are not multiplicative principal ones.

Definition 3.15 (cf. 3.13 of [5]) Let $\tau \in \mathbb{E}_{1}$ and $\alpha \in\left[\tau, \tau^{\infty}\right) \cap \mathbb{P}$. The tracking sequence of $\alpha$ above $\tau$, $\operatorname{ts}^{\tau}(\alpha)$, is defined as in Definition 3.5 if $\alpha \in \mathbb{M}^{>\tau}$, and otherwise recursively in the multiplicative decomposition of $\alpha$ as follows. 
1. If $\alpha \leq \tau^{\omega}$ then $\operatorname{ts}^{\tau}(\alpha):=(\alpha)$.

2. Otherwise. Then $\bar{\alpha} \in[\tau, \alpha)$ and $\alpha=_{\mathrm{NF}} \bar{\alpha} \cdot \beta$ for some $\beta \in \mathbb{M}^{>1}$. Let $\operatorname{ts}^{\tau}(\bar{\alpha})=\left(\alpha_{1}, \ldots, \alpha_{n}\right)$ and set $\alpha_{0}:=\tau 2$

2.1. If $\alpha_{n} \in \mathbb{E}^{>\alpha_{n-1}}$ and $\beta \leq \mu_{\alpha_{n}}^{\tau}$ then $\operatorname{ts}^{\tau}(\alpha):=\left(\alpha_{1}, \ldots, \alpha_{n}, \beta\right)$.

2.2. Otherwise. For $i \in\{1, \ldots, n\}$ let $\left(\beta_{1}^{i}, \ldots, \beta_{m_{i}}^{i}\right)$ be $\operatorname{ts}^{\alpha_{i}}(\beta)$ provided $\beta>\alpha_{i}$, and set $m_{i}:=1, \beta_{1}^{i}:=\alpha_{i} \cdot \beta$ if $\beta \leq \alpha_{i}$. We first define the critical index

$$
i_{0}(\alpha)=i_{0}:=\max \left(\{1\} \cup\left\{j \in\{2, \ldots, n\} \mid \beta_{1}^{j} \leq \mu_{\alpha_{j-1}}^{\tau}\right\}\right) .
$$

Then $\operatorname{ts}^{\tau}(\alpha):=\left(\alpha_{1}, \ldots, \alpha_{i_{0}-1}, \beta_{1}^{i_{0}}, \ldots, \beta_{m_{i_{0}}}^{i_{0}}\right)$.

Instead of $\operatorname{ts}^{1}(\alpha)$ we also simply write $\operatorname{ts}(\alpha)$.

Lemma 3.16 If in the above definition, part 2.2, we have $\beta>\alpha_{i_{0}}$, then for all $j \in\left(i_{0}, \ldots, n\right]$ we have

$$
\beta_{1}^{i_{0}} \leq \alpha_{j} \leq \mu_{\alpha_{j-1}}
$$

in particular $\beta_{1}^{i_{0}} \leq \mu_{\alpha_{i_{0}}}$.

Proof. Assume toward contradiction that there exists the maximal $j \in\left(i_{0}, \ldots, n\right]$ such that $\alpha_{j}<\beta_{1}^{i_{0}}$. Since $\beta_{1}^{i_{0}} \leq \beta \leq \alpha_{n}$ we have $j<n$ and obtain

$$
\alpha_{i_{0}}<\alpha_{j}<\beta_{1}^{i_{0}} \leq \alpha_{j+1} \leq \mu_{\alpha_{j}}
$$

implying that $\alpha_{j} \in \operatorname{ts}^{\alpha_{i_{0}}}(\beta)$, contradicting the minimality of $\beta_{1}^{i_{0}}$ in $\operatorname{ts}^{\alpha_{i_{0}}}(\beta)$.

Lemma 3.17 (3.14 of [5]) Let $\tau \in \mathbb{E}_{1}$ and $\alpha \in\left[\tau, \tau^{\infty}\right) \cap \mathbb{P}$. Let further $\left(\alpha_{1}, \ldots, \alpha_{n}\right)$ be $\operatorname{ts}^{\tau}(\alpha)$, the tracking sequence of $\alpha$ above $\tau$.

1. If $\alpha \in \mathbb{M}$ then $\alpha_{n}=\alpha$ and $\operatorname{ts}^{\tau}\left(\alpha_{i}\right)=\left(\alpha_{1}, \ldots, \alpha_{i}\right)$ for $i=1, \ldots, n$.

2. If $\alpha=_{\mathrm{NF}} \eta \cdot \xi \notin \mathbb{M}$ then $\alpha_{n} \in \mathbb{P} \cap[\xi, \alpha]$ and $\alpha_{n}={ }_{\mathrm{NF}} \overline{\alpha_{n}} \cdot \xi$.

3. $\left(\alpha_{1}, \ldots, \alpha_{n-1}\right)$ is either empty or a strictly increasing sequence of epsilon numbers in the interval $(\tau, \alpha)$.

4. For $1 \leq i \leq n-1$ we have $\alpha_{i+1} \leq \mu_{\alpha_{i}}^{\tau}$, and if $\alpha_{i}<\alpha_{i+1}$ then $\left(\alpha_{1}, \ldots, \alpha_{i+1}\right)$ is a subsequence of the $\tau$-localization of $\alpha_{i+1}$.

Proof. The proof proceeds by straightforward induction along the definition of $\operatorname{ts}^{\tau}(\alpha)$, i.e. along the length of the $\tau$-localization of multiplicative principal numbers and the number of factors in the multiplicative decomposition of additive principal numbers. In part 4 Lemma 6.5 of [7] and the previous remark apply.

Lemma 3.18 (3.15 of [5]) Let $\tau \in \mathbb{E}_{1}$ and $\alpha, \gamma \in\left[\tau, \tau^{\infty}\right) \cap \mathbb{P}, \alpha<\gamma$. Then we have

$$
\operatorname{ts}^{\tau}(\alpha)<_{\operatorname{lex}} \operatorname{ts}^{\tau}(\gamma)
$$


induction along the inductive definition of $\operatorname{ts}^{\tau}(\alpha)$.

Theorem 3.19 For all $\alpha \in \mathbb{P} \cap 1^{\infty}$ we have

$$
\mathrm{o}(\operatorname{ts}(\alpha))=\alpha .
$$

${ }^{2}$ As verified in part 2 of the lemma below we have $\beta \leq \alpha_{n}$. 
Proof. The theorem is proved by induction along the inductive definition of $\operatorname{ts}(\alpha)$.

Case 1: $\alpha \in \mathbb{M}$. Then $1 \operatorname{Seq}(\operatorname{ts}(\alpha))=(1, \ldots, 1)$ and hence $\mathrm{o}(\operatorname{ts}(\alpha))=\alpha$ immediately by definition.

Case 2: $\alpha={ }_{\mathrm{NF}} \bar{\alpha} \cdot \beta \in \mathbb{P}-\mathbb{M}$. Let $\operatorname{ts}(\bar{\alpha})=:\left(\alpha_{1}, \ldots, \alpha_{n}\right)$ and $\alpha_{0}:=1$. By the i.h. $o(\boldsymbol{\alpha})=\bar{\alpha}$. We have $\beta \leq \operatorname{lf}\left(\alpha_{n}\right) \leq \alpha_{n}, n \geq 1$.

Subcase 2.1: $\alpha_{n} \in \mathbb{E}^{>\alpha_{n-1}} \& \beta \leq \mu_{\alpha_{n}}$. Then $\operatorname{ts}(\alpha)=\boldsymbol{\alpha} \frown \beta$, and since $\beta \in \mathbb{M} \leq \alpha_{n}$ according to the definition of o we obtain $\mathrm{o}(\boldsymbol{\alpha} \frown \beta)=\mathrm{o}(\boldsymbol{\alpha}) \cdot \beta=\bar{\alpha} \cdot \beta=\alpha$.

Subcase 2.2: Otherwise. Let $\left(\beta_{1}^{i}, \ldots, \beta_{m_{i}}^{i}\right)$ for $i=1, \ldots, n$ as well as the index $i_{0}$ be defined as in case 2.2 of Definition 3.15] so that $\operatorname{ts}(\alpha)=\left(\alpha_{1}, \ldots, \alpha_{i_{0}-1}, \beta_{1}^{i_{0}}, \ldots, \beta_{m_{i_{0}}}^{i_{0}}\right)$.

2.2.1: $i_{0}=n$. Then we have $\operatorname{ts}(\alpha)=\left(\alpha_{1}, \ldots, \alpha_{n-1}, \alpha_{n} \cdot \beta\right)$, and using the i.h. we obtain $\mathrm{o}(\operatorname{ts}(\alpha))=\mathrm{o}(\boldsymbol{\alpha}) \cdot \beta=\alpha$.

2.2.2: $i_{0}<n$ and $\beta \leq \alpha_{i_{0}}$. Then we have $\alpha_{i_{0}} \in \mathbb{E}^{>\alpha_{i_{0}-1}}, \alpha_{i_{0}} \cdot \beta \leq \mu_{\alpha_{i_{0}-1}}$, and $\operatorname{ts}(\alpha)=\left(\alpha_{1}, \ldots, \alpha_{i_{0}-1}, \alpha_{i_{0}} \cdot \beta\right)$. It follows that for all $j \in\left(i_{0}, n\right]$ we have $\beta \leq \alpha_{j}$ and $\alpha_{j} \cdot \beta>\mu_{\alpha_{j-1}}$, hence $\beta \leq \mu_{\alpha_{i_{0}}}$ and thus

$$
\mathrm{o}(\operatorname{ts}(\alpha))=\mathrm{o}\left(\mathrm{h}_{\beta}\left(\boldsymbol{\alpha}_{\uparrow_{i_{0}}}\right)\right) \cdot \beta \text {. }
$$

The sequence $\mathrm{h}_{\beta}\left(\boldsymbol{\alpha}_{\uparrow_{i_{0}}}\right)$ is of the form $\boldsymbol{\alpha}_{\uparrow_{i_{0}-1}}-\boldsymbol{\delta}$ where $\boldsymbol{\delta}:=\operatorname{sk}_{\beta}\left(\alpha_{i_{0}}\right)$. Since $\mu_{\alpha_{i_{0}}}<\alpha_{i_{0}+1} \cdot \beta$ we have $\overline{\mu_{\alpha_{i_{0}}} \cdot \beta}=$ $\alpha_{i_{0}+1}=\delta_{2}$. In the case $i_{0}+1=n$ we obtain $\boldsymbol{\delta}=\left(\alpha_{n-1}, \alpha_{n}\right)$, hence $\mathrm{h}_{\beta}\left(\boldsymbol{\alpha}_{\uparrow_{i_{0}}}\right)=\boldsymbol{\alpha}$, otherwise we iterate the above argumentation to see that $\boldsymbol{\delta}=\left(\alpha_{i_{0}}, \ldots, \alpha_{n}\right)$. Hence $\mathrm{o}(\operatorname{ts}(\alpha))=\mathrm{o}(\boldsymbol{\alpha}) \cdot \beta$ as desired.

2.2.3: $i_{0}<n$ and $\alpha_{i_{0}}<\beta$. Then we have $\boldsymbol{\beta}^{i_{0}}=\operatorname{ts}^{\alpha_{i_{0}}}(\beta)$ with $\beta_{1}^{i_{0}} \leq \mu_{\alpha_{i_{0}-1}}$ if $i_{0}>1$. By Lemma 3.16 $\alpha_{i_{0}}$ is the immediate predecessor of $\beta_{1}^{i_{0}}$ in $\operatorname{ts}^{\alpha_{i_{0}-1}}(\beta)$. By definition of o we have $\mathrm{o}(\operatorname{ts}(\alpha))=\mathrm{o}\left(\mathrm{h}_{\beta}\left(\boldsymbol{\alpha}_{\uparrow_{i_{0}}}\right)\right) \cdot \beta$ and therefore have to show that $\mathrm{h}_{\beta}\left(\boldsymbol{\alpha}_{\uparrow_{i_{0}}}\right)=\boldsymbol{\alpha}$. We define

$$
j_{0}:=\min \left\{j \in\left\{i_{0}, \ldots, n-1\right\} \mid \beta \leq \mu_{\alpha_{j}}\right\},
$$

which exists, because $\beta \leq \alpha_{n} \leq \mu_{\alpha_{n-1}}$.

Claim: $\operatorname{mts}^{\alpha_{i_{0}}}(\beta)=\left(\alpha_{i_{0}}, \ldots, \alpha_{j_{0}}, \beta\right)$.

Proof. For every $j \in\left\{i_{0}, \ldots, j_{0}-1\right\}$ the minimality of $j_{0}$ implies $\beta>\mu_{\alpha_{j}} \geq \alpha_{j+1}$, and thus by the maximality of $i_{0}$ also $\beta_{1}^{j+1}>\mu_{\alpha_{j}}$. Moreover, we have

$$
\beta_{1}^{j+1} \leq \alpha_{j+2}:
$$

Assume otherwise and let $j$ be maximal in $\left\{i_{0}, \ldots, j_{0}-1\right\}$ such that $\beta_{1}^{j+1}>\alpha_{j+2}$. Since $\beta_{1}^{j+1} \leq \beta \leq \alpha_{n}$ we must have $j \leq n-3$. But then $\alpha_{j+1}<\alpha_{j+2}<\beta_{1}^{j+1} \leq \alpha_{j+3} \leq \mu_{j+2}$ and hence $\alpha_{j+2} \in \operatorname{ts}^{\alpha_{j+1}}(\beta)$, contradicting the minimality of $\beta_{1}^{j+1}$ in $\operatorname{ts}^{\alpha_{j+1}}(\beta)$. Therefore

$$
\mu_{\alpha_{j}}<\beta_{1}^{j+1} \leq \alpha_{j+2} \leq \mu_{\alpha_{j+1}}
$$

which concludes the proof of the claim.

It remains to be shown that $\operatorname{sk}_{\beta}\left(\alpha_{j_{0}}\right)=\left(\alpha_{j_{0}}, \ldots, \alpha_{n}\right)$, i.e. to successively check for $j=j_{0}, \ldots, n-1$ that $\beta \leq \alpha_{j+1} \leq \mu_{\alpha_{j}}$ and $\alpha_{j+1} \cdot \beta>\mu_{\alpha_{j}}$, whence $\overline{\mu_{\alpha_{j}} \cdot \beta}=\alpha_{j+1}$. This concludes the verification of $\mathrm{h}_{\beta}\left(\boldsymbol{\alpha}_{\uparrow_{i_{0}}}\right)=\boldsymbol{\alpha}$ and consequently the proof of the theorem.

Theorem 3.20 For all $\boldsymbol{\alpha} \sim \beta \in$ TS we have

$$
\operatorname{ts}\left(\mathrm{o}\left(\boldsymbol{\alpha}^{\frown} \beta\right)\right)=\boldsymbol{\alpha}^{\frown} \beta .
$$

Proof. The theorem is proved by induction on $\operatorname{lSeq}(\alpha \frown \beta)$ along the ordering (1Seq, $\left.<_{\text {lex }}\right)$. Let $\beta={ }_{\mathrm{NF}} \beta_{1} \cdot \ldots \cdot \beta_{k}$ and set $n_{0}:=n_{0}(\boldsymbol{\alpha} \frown \beta), \gamma:=\gamma(\boldsymbol{\alpha} \frown \beta)$ according to Definition 3.14, which provides us with an NF-representation of o $(\boldsymbol{\alpha} \frown \beta)$, where in the interesting cases the i.h. applies to the term ts $(\overline{\mathrm{o}(\boldsymbol{\alpha} \sim \beta)})$.

Case 1: $n=0$ and $\beta=1$. Trivial.

Case 2: $1<\beta_{1} \leq \alpha_{n}$. Then $\mathrm{o}(\boldsymbol{\alpha} \frown \beta)=_{\mathrm{NF}} \mathrm{o}(\boldsymbol{\alpha}) \cdot \beta$, and it is straightforward to verify the claim from the i.h. applied to $\boldsymbol{\alpha}$ by inspecting case 2.1 of Definition 3.15. 
Case 3: $k>1$ with $\beta_{1} \in \mathbb{E}^{>\alpha_{n}}$ and $\beta_{2} \leq \mu_{\beta_{1}}$. Then by definition $\mathrm{o}(\boldsymbol{\alpha} \frown \beta)=_{\mathrm{NF}} \mathrm{o}\left(\mathrm{h}_{\beta_{2}}\left(\boldsymbol{\alpha} \frown \beta_{1}\right)\right) \cdot \beta^{\prime}$ where $\beta^{\prime}=\left(1 / \beta_{1}\right) \cdot \beta$. According to part 2 of Definition 3.11 we have

$$
\mathrm{h}_{\beta_{2}}\left(\boldsymbol{\alpha} \frown \beta_{1}\right)=\boldsymbol{\alpha} \frown \boldsymbol{\delta}
$$

where $\boldsymbol{\delta}=\left(\delta_{1}, \ldots, \delta_{l+1}\right):=\operatorname{sk}_{\beta_{2}}\left(\beta_{1}\right)$. Assume first that $k=2$. Then the maximality of the length of $\boldsymbol{\delta}$ excludes the possibility $\delta_{l+1} \in \mathbb{E}^{>\delta_{l}} \& \beta_{2} \leq \mu_{\delta_{l+1}}$. We have $\beta_{2} \leq \beta_{1}$ and $\beta \leq \mu_{\alpha_{n}}$. For $j \in\{2, \ldots, l+1\}$ we have $\beta_{2} \leq \delta_{j}=\overline{\mu_{\delta_{j-1}} \cdot \beta_{2}} \leq \mu_{\delta_{j-1}}$ and $\delta_{j} \cdot \beta_{2}=\mu_{\delta_{j-1}} \cdot \beta_{2}>\mu_{\delta_{j-1}}$. This implies that $\operatorname{ts}(\mathrm{o}(\boldsymbol{\alpha} \frown \beta))=\boldsymbol{\alpha} \frown \beta$. The claim now follows easily for $k>2$ since $\beta \leq \mu_{\alpha_{n}}$.

Case 4: Otherwise.

Subcase 4.1: $n_{0}=0$. Then we have $\mathrm{o}(\boldsymbol{\alpha}-\beta)=\beta$, ts ${ }^{\alpha_{i-1}}\left(\alpha_{i}\right)=\left(\alpha_{i}\right)$ for $i=1, \ldots, n$, and $\operatorname{ts}^{\alpha_{n}}\left(\beta_{1}\right)=\left(\beta_{1}\right)$, whence $\operatorname{ts}(\beta)=(\beta)$.

Subcase 4.2: $n_{0}>0$. Using the abbreviation $\boldsymbol{\alpha}^{\prime}:=\boldsymbol{\alpha}_{\left\lceil n_{0}-1\right.}$ we then have $\mathrm{o}(\boldsymbol{\alpha} \frown \beta)=_{\mathrm{NF}} \mathrm{o}\left(\mathrm{h}_{\beta_{1}}\left(\boldsymbol{\alpha}^{\prime} \gamma \gamma\right)\right) \cdot \beta$. Setting $\operatorname{mts}^{\gamma}\left(\beta_{1}\right)=:\left(\gamma_{1}, \ldots, \gamma_{m+1}\right)$ where $\gamma_{1}=\gamma, \gamma_{m+1}=\beta_{1}$, and $\operatorname{sk}_{\beta_{1}}\left(\gamma_{m}\right)=: \boldsymbol{\delta}=\left(\delta_{1}, \ldots, \delta_{l+1}\right)$ where $\delta_{1}=\gamma_{m}$, we have

$$
\mathrm{h}_{\beta_{1}}\left(\boldsymbol{\alpha}^{\prime} \gamma\right)=\boldsymbol{\alpha}^{\prime} \gamma_{\uparrow_{m-1}} \frown \boldsymbol{\delta},
$$

which by the i.h. is the tracking sequence of $\overline{\mathrm{o}\left(\boldsymbol{\alpha} \frown \beta_{1}\right)}$. Assuming first that $k=1$, we now verify that the tracking sequence of o $(\boldsymbol{\alpha} \frown \beta)$ actually is $\boldsymbol{\alpha} \frown \beta$, by checking that case 2.2 of Definition 3.15 applies, with $n_{0}$ playing the role of the critical index $i_{0}(\mathrm{o}(\boldsymbol{\alpha}-\beta))$. Note first that the maximality of the length of $\boldsymbol{\delta}$ rules out the possibility $\delta_{l+1} \in \mathbb{E}^{>\delta_{l}} \& \beta_{1} \leq \mu_{\delta_{l+1}}$ and hence case 2.1 of Definition 3.15 According to the choice of $n_{0}$ and part 2 of Lemma 3.7 we have $\operatorname{ts}^{\gamma}\left(\beta_{1}\right)=\max -\operatorname{cov}^{\gamma}\left(\beta_{1}\right)=\left(\alpha_{n_{0}}, \ldots, \alpha_{n}, \beta_{1}\right)$ and of course $\alpha_{n_{0}} \leq \mu_{\alpha_{n_{0}-1}}$. Thus $n_{0}$ qualifies for the critical index, once we show its maximality: Firstly, for any $i \in\{2, \ldots, m\}$ we have $\gamma_{i}<\beta_{1}$, and setting $\boldsymbol{\beta}^{i}:=\operatorname{ts}^{\gamma_{i}}\left(\beta_{1}\right)$ the assumption $\beta_{1}^{i} \leq \mu_{\gamma_{i-1}}$ would imply that $\gamma_{\uparrow_{i-1}}-\boldsymbol{\beta}^{i}$ is a $\mu$-covering from $\gamma$ to $\beta_{1}$ such that

$$
\operatorname{mts}^{\gamma}\left(\beta_{1}\right)<_{\operatorname{lex}} \gamma_{\uparrow_{i-1}} \frown \boldsymbol{\beta}^{i}
$$

contradicting part 3 of Lemma 3.7. Secondly, for any $j \in\{2, \ldots, l+1\}$ we have $\delta_{j}=\overline{\mu_{\delta_{j-1}} \cdot \beta_{1}}$, so $\delta_{j} \cdot \beta_{1}=$ $\mu_{\delta_{j-1}} \cdot \beta_{1}>\mu_{\delta_{j-1}}$. These considerations entail

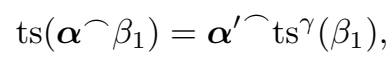

and it is easy now to verify the claim for arbitrary $k$, again since $\beta \leq \mu_{\alpha_{n}}$.

Corollary 3.21 o is strictly increasing with respect to the lexicographic ordering on TS and continuous in the last vector component.

Proof. The first statement is immediate from Lemma 3.18 and Theorems 3.19 and 3.20 . In order to verify continuity, let $\boldsymbol{\alpha}=\left(\alpha_{1}, \ldots, \alpha_{n}\right) \in \mathrm{RS}$ and $\beta \in \mathbb{L} \leq \mu_{\alpha_{n}}$ be given. For any $\gamma \in \mathbb{P} \cap \beta$, we have $\tilde{\gamma}:=\mathrm{o}(\boldsymbol{\alpha} \frown \gamma)<$ $\mathrm{o}(\boldsymbol{\alpha} \frown \beta)=: \tilde{\beta}$ and $\boldsymbol{\alpha}^{\frown} \gamma=\operatorname{ts}(\tilde{\gamma})<_{\operatorname{lex}} \operatorname{ts}(\tilde{\beta})=\boldsymbol{\alpha} \frown \beta$. For given $\tilde{\delta} \in \mathbb{P} \cap(\tilde{\gamma}, \tilde{\beta})$ set $\boldsymbol{\delta}:=\operatorname{ts}(\tilde{\delta})$, so that

$$
\boldsymbol{\alpha} \frown \gamma<_{\operatorname{lex}} \boldsymbol{\delta}<_{\operatorname{lex}} \boldsymbol{\alpha} \frown \beta,
$$

whence $\boldsymbol{\alpha} \subseteq \boldsymbol{\delta}$ is an initial segment. Writing $\boldsymbol{\delta}=\boldsymbol{\alpha} \frown\left(\zeta_{1}, \ldots, \zeta_{m}\right)$ we obtain $\gamma<\zeta_{1}<\beta$. For $\boldsymbol{\delta}^{\prime}:=\boldsymbol{\alpha} \frown \zeta_{1} \cdot \omega$ we then have $\tilde{\tilde{\delta}}<\mathrm{o}\left(\boldsymbol{\delta}^{\prime}\right)<\tilde{\beta}$.

Remark. Theorems 3.19 and 3.20 establish Lemma 4.10 of [5] in a weak theory, adjusted to our redefinition of o. Its equivalence with the definition in [5] follows, since the definition of ts has not been modified. In the next section we will continue in this way in order to obtain suitable redefinitions of the $\kappa$ - and $\nu$-functions.

The following lemma will not be required in the sequel, but it has been included to further illuminate the approach.

Lemma 3.22 Let $\boldsymbol{\alpha} \frown \beta \in \mathrm{RS}, \gamma \in \mathbb{M} \cap(1, \widehat{\beta})$, and let $\boldsymbol{\tau} \frown \sigma \in \mathrm{TS}$ be such that $\boldsymbol{\alpha} \frown \beta \subseteq \boldsymbol{\tau} \frown \sigma$ and $\mathrm{h}_{\gamma}(\boldsymbol{\alpha} \frown \beta)<_{\text {lex }}$

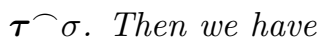

$$
\mathrm{o}\left(\boldsymbol{\tau}^{\frown} \sigma\right)=_{\mathrm{NF}} \mathrm{o}\left(\mathrm{h}_{\gamma}\left(\boldsymbol{\alpha}^{\frown} \beta\right)\right) \cdot \delta
$$

for some $\delta \in \mathbb{P} \cap(1, \gamma)$. 
Proof. The lemma is proved by induction on $\operatorname{Seq}\left(\boldsymbol{\tau}^{\frown} \sigma\right)$, using Lemma 3.12 In order to fix some notation, set $\boldsymbol{\tau}=\left(\tau_{1}, \ldots, \tau_{s}\right)$ and $\tau_{s+1}:=\sigma$. Write $\mathrm{h}_{\gamma}(\boldsymbol{\alpha} \frown \beta)=\boldsymbol{\alpha} \frown \boldsymbol{\eta} \frown \boldsymbol{\delta}$ where $\boldsymbol{\eta}=\left(\eta_{1}, \ldots, \eta_{r}\right)$ and $\boldsymbol{\delta}=\left(\delta_{1}, \ldots, \delta_{l+1}\right)=\operatorname{sk}_{\gamma}(\varepsilon)$, $\delta_{1}=\varepsilon=: \eta_{r+1}$, and $\delta_{l+2}:=1$. Note that since $\boldsymbol{\alpha} \frown \beta \in \mathrm{RS}$, we have $\varepsilon \in \mathbb{E}^{>\eta_{r}}$. According to Lemma 3.12 either one of the two following cases applies to $\tau^{\frown \sigma} \sigma$.

Case 1: $\boldsymbol{\tau}=\boldsymbol{\alpha}^{\frown} \boldsymbol{\eta} \frown \boldsymbol{\delta}_{\uparrow i}$ for some $i \in\{1, \ldots, l+1\}$ and $\sigma==_{\mathrm{NF}} \delta_{i+1} \cdot \zeta$ for some $\zeta \in \mathbb{P} \cap(1, \gamma)$. Let $\zeta=_{\mathrm{MNF}} \zeta_{1} \cdot \ldots \cdot \zeta_{j}$.

Subcase 1.1: $i=l+1$. Then $\mathrm{h}_{\gamma}(\boldsymbol{\alpha} \frown \beta)=\boldsymbol{\tau}, \delta_{l+1} \in \mathbb{E}^{>\delta_{l}}$, and $\sigma \leq \mu_{\delta_{l+1}}<\gamma \leq \delta_{l+1}$. According to the definition of o we have o $(\boldsymbol{\tau} \frown \sigma)=\mathrm{o}\left(\mathrm{h}_{\gamma}(\boldsymbol{\alpha} \frown \beta)\right) \cdot \sigma$.

Subcase 1.2: $i \leq l$ where $\delta_{i+1} \in \mathbb{E}^{>\delta_{i}}$ and $\zeta_{1} \leq \mu_{\delta_{i+1}}$. By definition, $\mathrm{o}(\boldsymbol{\tau} \frown \sigma)=\mathrm{o}\left(\mathrm{h}_{\zeta_{1}}\left(\boldsymbol{\tau} \frown \delta_{i+1}\right)\right) \cdot \zeta$. As $\boldsymbol{\tau}^{\frown} \delta_{i+1}$ is an initial segment of $\mathrm{h}_{\gamma}(\boldsymbol{\alpha} \frown \beta)$ and $\zeta_{1}<\gamma$, we have

$$
\mathrm{h}_{\gamma}(\boldsymbol{\alpha} \frown \beta)=\mathrm{h}_{\gamma}\left(\boldsymbol{\tau} \frown \delta_{i+1}\right) \leq_{\text {lex }} \mathrm{h}_{\zeta_{1}}\left(\tau \frown \delta_{i+1}\right)
$$

by Corollary 3.13 The claim now follows by the i.h. (if necessary) applied to $\mathrm{h}_{\zeta_{1}}\left(\tau \frown \delta_{i+1}\right)$.

Subcase 1.3: Otherwise. Here we must have $i=l$, since if $i<l$ it follows that $\delta_{i+1} \in \mathbb{E}^{>\delta_{i}}$ and $\zeta_{1}<$ $\gamma \leq \overline{\mu_{\delta_{i+1}} \cdot \gamma}=\delta_{i+2} \leq \mu_{\delta_{i+1}}$, which has been covered by the previous subcase. We therefore have $\mathrm{o}(\boldsymbol{\tau} \neg \sigma)=$ $\mathrm{o}\left(\mathrm{h}_{\gamma}(\boldsymbol{\alpha} \frown \beta)\right) \cdot \zeta$.

Case 2: $\boldsymbol{\tau}_{\uparrow_{s_{0}}}=\boldsymbol{\alpha}^{\frown} \boldsymbol{\eta}_{\uparrow_{r_{0}}}$ for some $r_{0} \in[1, r], s_{0} \leq s, \eta_{r_{0}+1}<\tau_{s_{0}+1}$, whence according to Lemma 3.12 we have $\sigma \leq \mu_{\tau_{s}}<\gamma, \mu_{\tau_{j}}<\gamma$ for $j=s_{0}+1, \ldots, s$, and $\mu_{\sigma}<\gamma$ if $\sigma \in \mathbb{E}^{>\tau_{s}}$. Let $\sigma={ }_{\mathrm{MNF}} \sigma_{1} \cdot \ldots \cdot \sigma_{k}$ and $\sigma^{\prime}:=\left(1 / \sigma_{1}\right) \cdot \sigma$.

In the case $s_{0}<s$ we have $\mathrm{h}_{\gamma}(\boldsymbol{\alpha} \sim \beta)<_{\operatorname{lex}} \boldsymbol{\tau}$, and noting that $\sigma<\gamma$, the i.h. straightforwardly applies to $\boldsymbol{\tau}$. Let us therefore assume that $s_{0}=s$, whence $\boldsymbol{\tau}=\boldsymbol{\alpha}^{\frown} \boldsymbol{\eta}_{\left.\right|_{r_{0}}}$ and $\eta_{r_{0}+1}<\sigma$. Then we have $\boldsymbol{\eta}^{\frown} \varepsilon<_{\text {lex }} \boldsymbol{\tau}^{\frown} \sigma$ and $\eta_{r_{0}}<\eta_{r_{0}+1}<\sigma \leq \mu_{\eta_{r_{0}}}<\gamma$.

Subcase 2.1: $k>1$ where $\sigma_{1} \in \mathbb{E}^{>\eta_{r_{0}}}$ and $\sigma_{2} \leq \mu_{\sigma_{1}}$. Then $\mathrm{o}(\boldsymbol{\tau} \frown \sigma)=\mathrm{o}\left(\mathrm{h}_{\sigma_{2}}\left(\boldsymbol{\tau} \frown \sigma_{1}\right)\right) \cdot \sigma^{\prime}$ and $\gamma>\sigma_{1} \geq \eta_{r_{0}+1} \in$ $\mathbb{E}^{>} \eta_{r_{0}}$. In the case $\sigma_{1}>\eta_{r_{0}+1}$ the i.h. applies to $\mathrm{h}_{\sigma_{2}}\left(\boldsymbol{\tau} \frown \sigma_{1}\right)$. Now assume $\sigma_{1}=\eta_{r_{0}+1}$. As in Subcase 1.2 we obtain

$$
\mathrm{h}_{\gamma}(\boldsymbol{\alpha} \frown \beta)=\mathrm{h}_{\gamma}\left(\boldsymbol{\tau}^{\frown} \sigma_{1}\right) \leq_{\operatorname{lex}} \mathrm{h}_{\sigma_{2}}\left(\boldsymbol{\tau}^{\frown} \sigma_{1}\right),
$$

and (if necessary) the i.h. applies to $\mathrm{h}_{\sigma_{2}}\left(\boldsymbol{\tau} \frown \sigma_{1}\right)$.

Subcase 2.2: Otherwise, i.e. Case 4 of Definition 3.14 applies. Let $n_{0}:=n_{0}\left(\boldsymbol{\tau}^{\frown} \sigma\right)$. We first assume that $k=1$, i.e. $\sigma \in \mathbb{M} \cap\left(\eta_{r_{0}+1}, \gamma\right)$.

2.2.1: $n_{0} \leq s$. We obtain $\mathrm{o}(\boldsymbol{\tau})=\xi \cdot \tau_{s}$ and $\mathrm{o}(\boldsymbol{\tau} \frown \sigma)=\xi \cdot \sigma$ where $\xi=1$ if $n_{0}=0$ and $\xi=\mathrm{h}_{\tau_{s}}\left(\boldsymbol{\tau}_{\left.\right|_{n_{0}-1}} \frown \gamma(\boldsymbol{\tau})\right)$. The $<,<_{\text {lex }}$-order isomorphism between TS and $\mathbb{P}^{<1^{\infty}}$ established by Lemma 3.18 and Theorems 3.19 and 3.20 yields

$$
\mathrm{o}(\boldsymbol{\tau})<\mathrm{o}\left(\mathrm{h}_{\gamma}(\boldsymbol{\alpha} \frown \beta)\right)<\mathrm{o}(\boldsymbol{\tau} \frown \sigma)
$$

and hence the claim.

2.2.2: $n_{0}=s+1$. We have $\sigma \in \mathbb{M} \cap\left(\eta_{r_{0}+1}, \gamma\right)$. Let $\zeta$ be the immediate predecessor of $\sigma$ in ts $\eta_{r_{0}}(\sigma)$. Then $\mathrm{o}(\boldsymbol{\tau} \frown \sigma)=\mathrm{o}\left(\mathrm{h}_{\sigma}(\boldsymbol{\tau} \frown \zeta)\right) \cdot \sigma$.

2.2.2.1: $\eta_{r_{0}+1}<\zeta$. Then the i.h. applies to $\tau \frown \zeta$.

2.2.2.2: $\eta_{r_{0}+1}=\zeta$. Then we argue as before, since

$$
\mathrm{h}_{\gamma}(\boldsymbol{\alpha} \frown \beta)=\mathrm{h}_{\gamma}\left(\boldsymbol{\tau} \frown \eta_{r_{0}+1}\right) \leq_{\text {lex }} \mathrm{h}_{\sigma}\left(\boldsymbol{\tau} \frown \eta_{r_{0}+1}\right),
$$

so that the i.h. (if necessary) applies to $\mathrm{h}_{\sigma}\left(\boldsymbol{\tau}^{\frown} \eta_{r_{0}+1}\right)$.

2.2.2.3: $\eta_{r_{0}+1}>\zeta$. Then $\zeta$ is an element of $\operatorname{ts}^{\eta_{r_{0}}}\left(\eta_{r_{0}+1}\right)$, and by a monotonicity argument as in 2.2 .1 we obtain the claim as a consequence of

$$
\mathrm{o}\left(\mathrm{h}_{\sigma}\left(\boldsymbol{\tau}^{\frown \zeta}\right)\right)<\mathrm{o}\left(\mathrm{h}_{\gamma}(\boldsymbol{\alpha} \frown \beta)\right)<\mathrm{o}\left(\boldsymbol{\tau}^{\frown} \sigma\right) .
$$

This concludes the proof for $k=1$, and for $k>1$ the claim now follows easily. 


\section{Enumerating relativized connectivity components}

Recall Definition 4.4 of [5]. We are now going to characterize the functions $\kappa$ and $\nu$ by giving an alternative definition which is considerably less intertwined. The first step is to define the restrictions of $\kappa^{\alpha}$ and $\nu^{\alpha}$ to additive principal indices. Recall part 3 of Lemma 2.3

Definition 4.1 Let $\boldsymbol{\alpha} \in \mathrm{RS}$ where $\boldsymbol{\alpha}=\left(\alpha_{1}, \ldots, \alpha_{n}\right), n \geq 0, \alpha_{0}:=1$. We define $\kappa_{\beta}^{\boldsymbol{\alpha}}$ and $\nu_{\beta}^{\boldsymbol{\alpha}}$ for additive principal $\beta$ as follows, writing $\kappa_{\beta}$ instead of $\kappa_{\beta}^{()}$.

Case 1: $n=0$. For $\beta<1^{\infty}$ define

$$
\kappa_{\beta}:=\mathrm{o}((\beta)) .
$$

Case 2: $n>0$. For $\beta \leq \mu_{\alpha_{n}}$, i.e. $\boldsymbol{\alpha} \sim \beta \in \mathrm{TS}$, define

$$
\nu_{\beta}^{\alpha}:=\mathrm{o}\left(\boldsymbol{\alpha}^{\frown} \beta\right) .
$$

$\kappa_{\beta}^{\boldsymbol{\alpha}}$ for $\beta \leq \lambda_{\alpha_{n}}$ is defined by cases. If $\beta \leq \alpha_{n}$ let $i \in\{0, \ldots, n-1\}$ be maximal such that $\alpha_{i}<\beta$. If $\beta>\alpha_{n}$ let $\beta={ }_{\mathrm{MNF}} \beta_{1} \cdot \ldots \cdot \beta_{k}$ and set $\beta^{\prime}:=\left(1 / \beta_{1}\right) \cdot \beta$.

$$
\kappa_{\beta}^{\boldsymbol{\alpha}}:= \begin{cases}\kappa_{\beta}^{\boldsymbol{\alpha}_{\uparrow_{i}}} & \text { if } \beta \leq \alpha_{n} \\ \mathrm{o}(\boldsymbol{\alpha}) \cdot \beta^{\prime} & \text { if } \beta_{1}=\alpha_{n} \& k>1 \\ \mathrm{o}(\boldsymbol{\alpha}-\beta) & \text { if } \beta_{1}>\alpha_{n} .\end{cases}
$$

Remark. Note that in the case $n>0$ we have the following inequalities between $\kappa_{\beta}^{\alpha}$ and $\nu_{\beta}^{\alpha}$, which are consequences of the monotonicity of o proved in Theorems 3.19 and 3.20

1. If $\beta \leq \alpha_{n}$ then $\kappa_{\beta}^{\boldsymbol{\alpha}} \leq \kappa_{\boldsymbol{\alpha}_{n} \mid \mathrm{n}-1}^{\boldsymbol{\alpha}}=\mathrm{o}(\boldsymbol{\alpha})$. Later we will define $\nu_{0}^{\boldsymbol{\alpha}}:=\mathrm{o}(\boldsymbol{\alpha})$.

2. If $\beta_{1}=\alpha_{n}$ and $k>1$ then $\kappa_{\beta}^{\alpha}=\mathrm{o}(\boldsymbol{\alpha}) \cdot \beta^{\prime}=\nu_{\beta^{\prime}}^{\boldsymbol{\alpha}}$, which is less than $\nu_{\beta}^{\boldsymbol{\alpha}}$ if $\beta \leq \mu_{\alpha_{n}}$.

3. Otherwise we have $\kappa_{\beta}^{\alpha}=\nu_{\beta}^{\alpha}$.

\section{Corollary 4.2}

1. $\kappa$ and $\nu$ are strictly increasing with respect to their $<_{\text {lex }}$-ordered arguments $\boldsymbol{\alpha}-\beta \in \mathrm{TS}$.

2. Each branch $\kappa^{\boldsymbol{\alpha}}$ (where $\boldsymbol{\alpha} \in \mathrm{RS}$ ) and $\nu^{\boldsymbol{\alpha}}$ (where $\left.\boldsymbol{\alpha} \in \mathrm{RS}-\{()\}\right)$ is continuous at arguments $\beta \in \mathbb{L}$.

Proof. This is a consequence of Corollary 3.21

We now prepare for the conservative extension of $\kappa$ and $\nu$ to their entire domain as well as the definition of dp which is in accordance with Definition 4.4 of [5].

Definition 4.3 Let $\boldsymbol{\tau} \in \mathrm{RS}, \boldsymbol{\tau}=\left(\tau_{1}, \ldots, \tau_{n}\right), n \geq 0, \tau_{0}:=1$. The term system $\mathrm{T}^{\boldsymbol{\tau}}$ is obtained from $\mathrm{T}^{\tau_{n}}$ by successive substitution of parameters in $\left(\tau_{i}, \tau_{i+1}\right)$ by their $\mathrm{T}^{\tau_{i}}$-representations, for $i=n-1, \ldots, 1$. The parameters $\tau_{i}$ are represented by the terms $\vartheta^{\tau_{i}}(0)$. The length $\mathrm{I}^{\boldsymbol{\tau}}(\alpha)$ of a $\mathrm{T}^{\tau}$-term $\alpha$ is defined inductively by

1. $\mathrm{l}^{\tau}(0):=0$,

2. $\mathrm{l}^{\boldsymbol{\tau}}(\beta):=\mathrm{l}^{\boldsymbol{\tau}}(\gamma)+\mathrm{l}^{\boldsymbol{\tau}}(\delta)$ if $\beta==_{\mathrm{NF}} \gamma+\delta$, and

3. $\mathrm{l}^{\boldsymbol{\tau}}(\vartheta(\eta)):=\left\{\begin{array}{lll}1 & \text { if } & \eta=0 \\ \mathrm{l}^{\boldsymbol{\tau}}(\eta)+4 & \text { if } & \eta>0\end{array}\right.$

where $\vartheta \in\left\{\vartheta^{\tau_{i}} \mid 0 \leq i \leq n\right\} \cup\left\{\vartheta_{i+1} \mid i \in \mathbb{N}\right\}$.

Remark. Recall Equation (11) as well as Lemma 2.13 and Definitions 2.14 and 2.18 of [9].

1. For $\beta=\vartheta^{\tau_{n}}(\Delta+\eta) \in \mathbb{E}$ such that $\beta \leq \mu_{\tau_{n}}$ we have

$$
\mathrm{l}^{\boldsymbol{\tau}}(\Delta)=\mathrm{l}^{\boldsymbol{\tau}^{-} \beta}\left(\iota_{\tau_{n}, \beta}(\Delta)\right)<\mathrm{l}^{\boldsymbol{\tau}}(\beta) .
$$


2. For $\beta \in \mathrm{T}^{\boldsymbol{\tau}} \cap \mathbb{P}^{>1} \cap \Omega_{1}$ let $\tau \in\left\{\tau_{0}, \ldots, \tau_{n}\right\}$ be maximal such that $\tau<\beta$. Clearly,

$$
\mathrm{l}^{\boldsymbol{\tau}}(\bar{\beta})<\mathrm{l}^{\boldsymbol{\tau}}(\beta)
$$

cf. Subsection 2.3, and

$$
\mathrm{l}^{\boldsymbol{\tau}}\left(\zeta_{\beta}^{\tau}\right)<\mathrm{l}^{\boldsymbol{\tau}}(\beta)
$$

In case of $\beta \notin \mathbb{E}$ we have

$$
\mathrm{l}^{\boldsymbol{\tau}}(\log (\beta)), \mathrm{l}^{\boldsymbol{\tau}}(\log ((1 / \tau) \cdot \beta))<\mathrm{l}^{\boldsymbol{\tau}}(\beta)
$$

and for $\beta \in \mathbb{E}$ we have

$$
\mathrm{l}^{\tau \frown \beta}\left(\lambda_{\beta}^{\tau}\right)<\mathrm{l}^{\boldsymbol{\tau}}(\beta)
$$

Finally, the definition of the enumeration functions of relativized connectivity components can be completed. This is easily seen to be a sound, elementary recursive definition.

Definition 4.4 (cf. 4.4 of [5]) Let $\boldsymbol{\alpha} \in \mathrm{RS}$ where $\boldsymbol{\alpha}=\left(\alpha_{1}, \ldots, \alpha_{n}\right), n \geq 0$, and set $\alpha_{0}:=1$. We define the functions

$$
\kappa^{\alpha}, \operatorname{dp}_{\alpha}: \operatorname{dom}\left(\kappa^{\alpha}\right) \rightarrow 1^{\infty}
$$

where $\operatorname{dom}\left(\kappa^{\boldsymbol{\alpha}}\right):=1^{\infty}$ if $n=0$ and $\operatorname{dom}\left(\kappa^{\boldsymbol{\alpha}}\right):=\left[0, \lambda_{\alpha_{n}}\right]$ if $n>0$, simultaneously by recursion on $1^{\boldsymbol{\alpha}}(\beta)$, extending Definition 4.1. The clauses extending the definition of $\kappa^{\alpha}$ are as follows.

1. $\kappa_{0}^{\alpha}:=0, \kappa_{1}^{\alpha}:=1$,

2. $\kappa_{\beta}^{\boldsymbol{\alpha}}:=\kappa_{\gamma}^{\boldsymbol{\alpha}}+\operatorname{dp}_{\boldsymbol{\alpha}}(\gamma)+\kappa_{\delta}^{\boldsymbol{\alpha}}$ for $\beta={ }_{\mathrm{NF}} \gamma+\delta$.

$\mathrm{dp}_{\boldsymbol{\alpha}}$ is defined as follows, using $\nu$ as already defined on TS.

1. $\operatorname{dp}_{\boldsymbol{\alpha}}(0):=0, \operatorname{dp}_{\boldsymbol{\alpha}}(1):=0$, and $\operatorname{dp}_{\boldsymbol{\alpha}}\left(\alpha_{n}\right):=0$ in case of $n>0$,

2. $\operatorname{dp}_{\boldsymbol{\alpha}}(\beta):=\operatorname{dp}_{\boldsymbol{\alpha}}(\delta)$ if $\beta={ }_{\mathrm{NF}} \gamma+\delta$,

3. $\operatorname{dp}_{\boldsymbol{\alpha}}(\beta):=\operatorname{dp}_{\boldsymbol{\alpha}\lceil n-1}(\beta)$ if $n>0$ for $\beta \in \mathbb{P} \cap\left(1, \alpha_{n}\right)$,

4. for $\beta \in \mathbb{P}^{>\alpha_{n}}-\mathbb{E}$ let $\gamma:=\left(1 / \alpha_{n}\right) \cdot \beta$ and $\log (\gamma)={ }_{\mathrm{ANF}} \gamma_{1}+\ldots+\gamma_{m}$ and set

$$
\operatorname{dp}_{\boldsymbol{\alpha}}(\beta):=\kappa_{\gamma_{1}}^{\boldsymbol{\alpha}}+\operatorname{dp}_{\boldsymbol{\alpha}}\left(\gamma_{1}\right)+\ldots+\kappa_{\gamma_{m}}^{\boldsymbol{\alpha}}+\operatorname{dp}_{\boldsymbol{\alpha}}\left(\gamma_{m}\right)
$$

5. for $\beta \in \mathbb{E}^{>\alpha_{n}}$ let $\gamma:=\left(\alpha_{1}, \ldots, \alpha_{n}, \beta\right)$, and set

$$
\operatorname{dp}_{\boldsymbol{\alpha}}(\beta):=\nu_{\mu_{\beta}^{\alpha_{n}}}^{\gamma}+\kappa_{\lambda_{\beta}^{\alpha_{n}}}^{\gamma}+\operatorname{dp}_{\gamma}\left(\lambda_{\beta}^{\alpha_{n}}\right)
$$

Definition 4.5 (cf. 4.4 of [5]) Let $\boldsymbol{\alpha} \in \mathrm{RS}$ where $\boldsymbol{\alpha}=\left(\alpha_{1}, \ldots, \alpha_{n}\right), n>0$, and set $\alpha_{0}:=1$. We define

$$
\nu^{\boldsymbol{\alpha}}: \operatorname{dom}\left(\nu^{\boldsymbol{\alpha}}\right) \rightarrow 1^{\infty}
$$

where $\operatorname{dom}\left(\nu^{\boldsymbol{\alpha}}\right):=\left[0, \mu_{\alpha_{n}}\right]$, extending Definition [4.1 and setting $\alpha:=\mathrm{o}(\boldsymbol{\alpha})$, by

1. $\nu_{0}^{\alpha}:=\alpha$,

2. $\nu_{\beta}^{\boldsymbol{\alpha}}:=\nu_{\gamma}^{\boldsymbol{\alpha}}+\kappa_{\varrho_{\gamma}^{\alpha_{n}}}^{\boldsymbol{\alpha}}+\operatorname{dp}_{\boldsymbol{\alpha}}\left(\varrho_{\gamma}^{\alpha_{n}}\right)+\check{\chi}^{\alpha_{n}}(\gamma) \cdot \alpha$ if $\beta=\gamma+1$,

3. $\nu_{\beta}^{\boldsymbol{\alpha}}:=\nu_{\gamma}^{\boldsymbol{\alpha}}+\kappa_{\varrho_{\gamma}^{\alpha_{n}}}^{\boldsymbol{\alpha}}+\operatorname{dp}_{\boldsymbol{\alpha}}\left(\varrho_{\gamma}^{\alpha_{n}}\right)+\nu_{\delta}^{\boldsymbol{\alpha}}$ if $\beta={ }_{\mathrm{NF}} \gamma+\delta \in \operatorname{Lim}$.

In the sequel we want to establish the results of Lemma 4.5 of [5] for the new definitions within a weak theory, avoiding the long transfinite induction used in the corresponding proof in [5]. Then the agreement of the definitions of $\kappa, \mathrm{dp}$ and $\nu$ in [5] and here can be shown in a weak theory as well. This includes also Lemma 4.7 of [5] and extends to the relativization of tracking sequences to contexts as lined out in Definition 4.13 through Lemma 4.17 of $[5]$. 
Lemma 4.6 Let $\boldsymbol{\alpha}=\left(\alpha_{1}, \ldots, \alpha_{n}\right) \in \mathrm{RS}$ and set $\alpha_{0}:=1$.

1. Let $\gamma \in \operatorname{dom}\left(\kappa^{\alpha}\right) \cap \mathbb{P}$. If $\gamma={ }_{\mathrm{MNF}} \gamma_{1} \cdot \ldots \cdot \gamma_{k} \geq \alpha_{n}$, setting $\gamma^{\prime}:=\left(1 / \gamma_{1}\right) \cdot \gamma$, we have

$$
\left(\kappa_{\gamma}^{\boldsymbol{\alpha}}+\operatorname{dp}_{\boldsymbol{\alpha}}(\gamma)\right) \cdot \omega=\left\{\begin{array}{cc}
\mathrm{o}(\boldsymbol{\alpha}) \cdot \gamma^{\prime} \cdot \omega & \text { if } \gamma_{1}=\alpha_{n} \\
\mathrm{o}(\boldsymbol{\alpha}-\gamma \cdot \omega) & \text { otherwise. }
\end{array}\right.
$$

If $\gamma<\alpha_{n}$ we have $\left(\kappa_{\gamma}^{\boldsymbol{\alpha}}+\operatorname{dp}_{\boldsymbol{\alpha}}(\gamma)\right) \cdot \omega<\mathrm{o}(\boldsymbol{\alpha})$.

2. For $\gamma \in \operatorname{dom}\left(\kappa^{\alpha}\right)-(\mathbb{E} \cup\{0\})$ we have

$$
\operatorname{dp}_{\boldsymbol{\alpha}}(\gamma)<\kappa_{\gamma}^{\boldsymbol{\alpha}}
$$

3. For $\gamma \in \mathbb{E}^{>\alpha_{n}}$ such that $\mu_{\gamma}<\gamma$ we have

$$
\operatorname{dp}_{\boldsymbol{\alpha}}(\gamma)<\mathrm{o}(\boldsymbol{\alpha} \frown \gamma) \cdot \mu_{\gamma} \cdot \omega
$$

4. For $\gamma \in \operatorname{dom}\left(\kappa^{\boldsymbol{\alpha}}\right) \cap \mathbb{E}^{>\alpha_{n}}$ we have

$$
\kappa_{\gamma}^{\boldsymbol{\alpha}} \cdot \omega \leq \operatorname{dp}_{\boldsymbol{\alpha}}(\gamma) \quad \text { and } \quad \operatorname{dp}_{\boldsymbol{\alpha}}(\gamma) \cdot \omega==_{\mathrm{NF}} \mathrm{o}\left(\mathrm{h}_{\omega}\left(\boldsymbol{\alpha}^{\frown} \gamma\right)\right) \cdot \omega .
$$

5. Let $\gamma \in \operatorname{dom}\left(\nu^{\alpha}\right) \cap \mathbb{P}, \gamma={ }_{\mathrm{MNF}} \gamma_{1} \cdot \ldots \cdot \gamma_{k}$. We have

$$
\left(\nu_{\gamma}^{\boldsymbol{\alpha}}+\kappa_{\varrho_{\gamma}^{\alpha_{n}}}^{\boldsymbol{\alpha}_{n}}+\operatorname{dp}_{\boldsymbol{\alpha}}\left(\varrho_{\gamma}^{\alpha_{n}}\right)\right) \cdot \omega= \begin{cases}\mathrm{o}(\boldsymbol{\alpha}) \cdot \gamma \cdot \omega & \text { if } \gamma_{1} \leq \alpha_{n} \\ \mathrm{o}\left(\mathrm{h}_{\omega}\left(\boldsymbol{\alpha}^{\frown} \gamma\right)\right) \cdot \omega & \text { if } \gamma \in \mathbb{E}^{>\alpha_{n}} \\ \mathrm{o}\left(\boldsymbol{\alpha}^{\frown} \gamma\right) \cdot \omega & \text { otherwise. }\end{cases}
$$

Proof. The lemma is shown by simultaneous induction on $1^{\alpha}(\gamma)$ over all parts.

Ad 1. The claim is immediate if $\gamma=\alpha_{n}$, and if $\gamma_{1}=\alpha_{n}$ and $k>1$, we have $\kappa_{\gamma}^{\boldsymbol{\alpha}}=\mathrm{o}(\boldsymbol{\alpha}) \cdot \gamma^{\prime}$ and by part 2 the claim follows. Now assume that $\gamma_{1}>\alpha_{n}$, whence $\kappa_{\gamma}^{\boldsymbol{\alpha}}=\mathrm{o}(\boldsymbol{\alpha} \frown \gamma)$. The case $\gamma \notin \mathbb{E}$ is handled again by part 2 . If $\gamma \in \mathbb{E}$, we apply part 4 to see that

$$
\left(\kappa_{\gamma}^{\boldsymbol{\alpha}}+\operatorname{dp}_{\boldsymbol{\alpha}}(\gamma)\right) \cdot \omega=\mathrm{dp}_{\boldsymbol{\alpha}}(\gamma) \cdot \omega=\mathrm{o}\left(\mathrm{h}_{\omega}\left(\boldsymbol{\alpha}^{\frown} \gamma\right)\right) \cdot \omega,
$$

and since $\mu_{\gamma} \geq \omega$, the latter is equal to o $(\boldsymbol{\alpha} \frown \gamma \cdot \omega)$.

Now consider the situation where $\gamma<\alpha_{n}$. Let $i \in[0, \ldots, n-1]$ be maximal such that $\alpha_{i}<\gamma$. The same argument as above yields the corresponding claim for $\boldsymbol{\alpha}_{\uparrow_{i}}$ instead of $\boldsymbol{\alpha}$, and by the monotonicity of o we see that the resulting ordinal is strictly below o $\left(\boldsymbol{\alpha}_{\uparrow_{i+1}}\right) \leq \mathrm{o}(\boldsymbol{\alpha})$.

Note that in the case $\gamma \cdot \omega \in \operatorname{dom}\left(\kappa^{\boldsymbol{\alpha}}\right)$ we have $\left(\kappa_{\gamma}^{\boldsymbol{\alpha}}+\operatorname{dp}_{\boldsymbol{\alpha}}(\gamma)\right) \cdot \omega=\kappa_{\gamma \cdot \omega}^{\boldsymbol{\alpha}}$ as a direct consequence of the definitions.

Ad 2. We may assume that $\gamma>\alpha_{n}$ (otherwise replace $n$ by the suitable $i<n$ and $\boldsymbol{\alpha}$ by $\boldsymbol{\alpha}_{\Gamma_{i}}$ ). Set $\gamma^{\prime}:=$ $\log \left(\left(1 / \alpha_{n}\right) \cdot \gamma\right)==_{\mathrm{ANF}} \gamma_{1}+\ldots+\gamma_{m}$, so that $\gamma=\alpha_{n} \cdot \omega^{\gamma_{1}+\ldots+\gamma_{m}}$ and $\operatorname{dp}_{\boldsymbol{\alpha}}(\gamma)=\sum_{i=1}^{m}\left(\kappa_{\gamma_{i}}^{\boldsymbol{\alpha}}+\operatorname{dp}_{\boldsymbol{\alpha}}\left(\gamma_{i}\right)\right)$. According to the definition, $\kappa_{\gamma}^{\boldsymbol{\alpha}}$ is either $\mathrm{o}(\boldsymbol{\alpha}) \cdot \omega^{\gamma_{1}+\ldots+\gamma_{m}}$ if $\gamma_{1} \leq \alpha_{n}$, or o $\left(\boldsymbol{\alpha}^{\frown} \gamma\right)$ if $\gamma_{1}>\alpha_{n}$. In the case $\gamma_{i} \cdot \omega<\gamma$ for $i=1, \ldots, m$ an application of part 1 of the i.h. to the $\gamma_{i}$ yields the claim, thanks to the monotonicity of o. Otherwise we must have $\gamma=\gamma_{1} \cdot \omega$ where $\gamma_{1} \in \mathbb{E}^{>\alpha_{n}}$, and applying part 1 of the i.h. to $\gamma_{1}$ yields

$$
\operatorname{dp}_{\boldsymbol{\alpha}}(\gamma)=\kappa_{\gamma_{1}}^{\boldsymbol{\alpha}}+\operatorname{dp}_{\boldsymbol{\alpha}}\left(\gamma_{1}\right)+1<\left(\kappa_{\gamma_{1}}^{\boldsymbol{\alpha}}+\operatorname{dp}_{\boldsymbol{\alpha}}\left(\gamma_{1}\right)\right) \cdot \omega=\mathrm{o}\left(\boldsymbol{\alpha}^{\frown} \gamma\right)=\kappa_{\gamma}^{\boldsymbol{\alpha}} .
$$

Ad 3. Let $\lambda_{\gamma}={ }_{\mathrm{ANF}} \lambda_{1}+\ldots+\lambda_{r}, \lambda \in\left\{\lambda_{1}, \ldots, \lambda_{r}\right\}$, and note that $\lambda \leq \gamma \cdot \mu_{\gamma}$. Setting $\lambda^{\prime}:=(1 / \operatorname{mf}(\lambda)) \cdot \lambda$, we have $\lambda^{\prime} \leq \mu_{\gamma}$ and applying part 1 of the i.h. to $\lambda$ we obtain $\left(\kappa_{\lambda}^{\boldsymbol{\alpha}}+\operatorname{dp}_{\boldsymbol{\alpha}}(\lambda)\right) \cdot \omega \leq \mathrm{o}(\boldsymbol{\alpha} \frown \gamma) \cdot \mu_{\gamma} \cdot \omega$. Since $\nu_{\mu_{\gamma}}^{\boldsymbol{\alpha}^{\smile} \gamma}=\mathrm{o}\left(\boldsymbol{\alpha}^{\frown}\left(\gamma, \mu_{\gamma}\right)\right)=\mathrm{o}\left(\boldsymbol{\alpha}^{\frown} \gamma\right) \cdot \mu_{\gamma}$, we obtain the claim.

Ad 4. The inequality is seen by a quick inspection of the respective definitions. We have $\kappa_{\gamma}^{\boldsymbol{\alpha}}=\mathrm{o}\left(\boldsymbol{\alpha}^{\frown} \gamma\right)$, and since $\mu_{\gamma} \geq \omega$ we obtain

$$
\mathrm{o}\left(\boldsymbol{\alpha}^{\frown} \gamma\right) \cdot \omega=\mathrm{o}(\boldsymbol{\alpha} \frown(\gamma, \omega)) \leq \mathrm{o}\left(\boldsymbol{\alpha} \frown\left(\gamma, \mu_{\gamma}\right)\right) \leq \operatorname{dp}_{\boldsymbol{\alpha}}(\gamma) .
$$

In order to verify the claimed equation, note that

$$
\mathrm{h}_{\omega}\left(\boldsymbol{\alpha}^{\frown} \gamma\right)=\boldsymbol{\alpha}^{\frown} \operatorname{sk}_{\omega}(\gamma)
$$


where $\operatorname{sk}_{\omega}(\gamma)=\left(\delta_{1}, \ldots, \delta_{l+1}\right)$ consists of a maximal strictly increasing chain $\delta:=\left(\delta_{1}, \ldots, \delta_{l}\right)=\left(\gamma, \mu_{\gamma}, \mu_{\mu_{\gamma}}, \ldots\right)$ of $\mathbb{E}$-numbers and $\delta_{l+1}=\mu_{\delta_{l}} \notin \mathbb{E}^{>\delta_{l}}$. We have $\lambda_{\delta_{i}}=\varrho_{\mu_{\delta_{i}}}+\zeta_{\delta_{i}}$ where $\zeta_{\delta_{i}}<\delta_{i}$ and, for $i<l, \varrho_{\mu_{\delta_{i}}}=\mu_{\delta_{i}}=\delta_{i+1} \in \mathbb{E}^{>\delta_{i}}$. Applying the i.h. to (the additive decompositions) of these terms $\lambda_{\delta_{i}}$ we obtain

$$
\operatorname{dp}_{\boldsymbol{\alpha}}(\gamma) \cdot \omega=\operatorname{dp}_{\boldsymbol{\alpha} \frown \boldsymbol{\delta}_{\vdash_{l-1}}}\left(\delta_{l}\right) \cdot \omega=\left(\nu_{\mu_{\delta_{l}}}^{\boldsymbol{\alpha} \boldsymbol{\delta}}+\kappa_{\lambda_{\delta_{l}}}^{\boldsymbol{\alpha} \frown \boldsymbol{\delta}}+\operatorname{dp}_{\boldsymbol{\alpha} \frown \boldsymbol{\delta}}\left(\lambda_{\delta_{l}}\right)\right) \cdot \omega
$$

and consider the additive decomposition of the term $\lambda_{\delta_{l}}$. The components below $\delta_{l}$ from $\zeta_{\delta_{l}}$ are easily handled using the inequality of part 1 of the i.h., while for $\mu_{\delta_{l}}={ }_{\mathrm{MNF}} \mu_{1} \cdot \ldots \cdot \mu_{j}$ we have $\varrho_{\mu_{\delta_{l}}} \leq \delta_{l} \cdot \log \left(\mu_{\delta_{l}}\right)=\delta_{l}$. $\left(\log \left(\mu_{1}\right)+\ldots+\log \left(\mu_{j}\right)\right)$ (where the only possible difference is $\delta_{l}$ ) and consider the summands separately. Let $\mu \in\left\{\mu_{1}, \ldots, \mu_{j}\right\}$.

Case 1: $\varrho_{\mu_{l}} \in \mathbb{E}^{>\delta_{l}}$. Let $\log \left(\mu_{\delta_{l}}\right)=\lambda+k$ where $\lambda \in \operatorname{Lim} \cup\{0\}$ and $k<\omega$. We must have $\chi^{\delta_{l}}(\lambda)=1$, since otherwise $\varrho_{\mu_{\delta_{l}}}=\mu_{\delta_{l}} \in \mathbb{E}^{>\delta_{l}}$, which would contradict the maximality of the length of $\boldsymbol{\delta}$. It follows that $k=1$, hence $\mu_{\delta_{l}}=\lambda \cdot \omega, \lambda=\varrho_{\delta_{\delta_{l}}} \in \mathbb{E}^{>\delta_{l}}$, and applying the i.h. to $\lambda$ yields

$$
\left(\kappa_{\lambda}^{\boldsymbol{\alpha} \frown \boldsymbol{\delta}}+\operatorname{dp}_{\boldsymbol{\alpha} \frown \boldsymbol{\delta}}(\lambda)\right) \cdot \omega=\mathrm{o}\left(\mathrm{h}_{\omega}(\boldsymbol{\alpha} \frown \boldsymbol{\delta} \frown \lambda)\right) \cdot \omega=\mathrm{o}\left(\boldsymbol{\alpha} \frown \boldsymbol{\delta}^{\frown \lambda} \lambda \omega\right)=\nu_{\mu_{\delta_{l}}}^{\boldsymbol{\alpha} \boldsymbol{\delta}}
$$

whence $\operatorname{dp}_{\boldsymbol{\alpha}}(\gamma) \cdot \omega=\mathrm{o}\left(\mathrm{h}_{\omega}(\boldsymbol{\alpha} \frown \gamma)\right) \cdot \omega$ as claimed.

Case 2: Otherwise.

Subcase 2.1: $\mu<\delta_{l}$. Then applying part 2 of the i.h. to $\delta_{l} \cdot \log (\mu)$ we see that

$$
\operatorname{dp}_{\boldsymbol{\alpha} \frown \boldsymbol{\delta}}\left(\delta_{l} \cdot \log (\mu)\right)<\kappa_{\delta_{l} \cdot \log (\mu)}^{\boldsymbol{\alpha} \frown \boldsymbol{\delta}}=\mathrm{o}(\boldsymbol{\alpha} \frown \boldsymbol{\delta}) \cdot \log (\mu) .
$$

Subcase 2.2: $\mu=\delta_{l}$. We calculate $\kappa_{\delta_{l}^{2}}^{\boldsymbol{\alpha}} \boldsymbol{\delta}=\mathrm{o}(\boldsymbol{\alpha} \frown \boldsymbol{\delta}) \cdot \delta_{l}$ and $\operatorname{dp}_{\boldsymbol{\alpha} \frown \boldsymbol{\delta}}\left(\delta_{l}^{2}\right)=\mathrm{o}(\boldsymbol{\alpha} \frown \boldsymbol{\delta})$.

Subcase 2.3: $\mu>\delta_{l}$.

2.3.1: $\mu \notin \mathbb{E}^{>\delta_{l}}$. Then $\delta_{l}<\delta_{l} \cdot \log (\mu) \notin \mathbb{E}^{>\delta_{l}}$, hence by the i.h., applied to $\delta_{l} \cdot \log (\mu)$, which is a summand of $\lambda_{\delta_{l}}$,

$$
\operatorname{dp}_{\boldsymbol{\alpha} \frown \boldsymbol{\delta}}\left(\delta_{l} \cdot \log (\mu)\right)<\kappa_{\delta_{l} \cdot \log (\mu)}^{\boldsymbol{\alpha} \boldsymbol{\delta}} \leq \nu_{\mu}^{\boldsymbol{\alpha} \frown \boldsymbol{\delta}} \leq \nu_{\mu_{\delta_{l}}}^{\boldsymbol{\alpha} \frown \boldsymbol{\delta}} .
$$

2.3.2: $\mu \in \mathbb{E}^{>\delta_{l}}$. Then we have $\delta_{l} \cdot \log (\mu)=\mu, \mu \cdot \omega \leq \mu_{\delta_{l}}$, and applying the i.h. to $\mu$

$$
\operatorname{dp}_{\boldsymbol{\alpha} \frown \boldsymbol{\delta}}(\mu) \cdot \omega=\mathrm{o}\left(\mathrm{h}_{\omega}\left(\boldsymbol{\alpha}^{\frown} \boldsymbol{\delta} \frown \mu\right)\right) \cdot \omega=\nu_{\mu \cdot \omega}^{\boldsymbol{\alpha}} \boldsymbol{\delta} \leq \nu_{\mu_{\delta_{l}}}^{\boldsymbol{\alpha} \boldsymbol{\delta}} .
$$

Ad 5. We have $\varrho_{\gamma} \leq \alpha_{n} \cdot\left(\log \left(\gamma_{1}\right)+\ldots+\log \left(\gamma_{k}\right)\right)$.

Case 1: $\gamma \in \mathbb{E}^{>\alpha_{n}}$. Here we have $\varrho_{\gamma}=\gamma$ and $\nu_{\gamma}^{\boldsymbol{\alpha}}=\kappa_{\gamma}^{\boldsymbol{\alpha}}<\operatorname{dp}_{\boldsymbol{\alpha}}(\gamma)=\nu_{\mu_{\gamma}}^{\boldsymbol{\alpha} \gamma}+\kappa_{\lambda_{\gamma}}^{\boldsymbol{\alpha} \gamma}+\operatorname{dp} \boldsymbol{\alpha}_{\gamma}\left(\lambda_{\gamma}\right)$, and by part 4 we have $\operatorname{dp}_{\boldsymbol{\alpha}}(\gamma) \cdot \omega=\mathrm{o}\left(\mathrm{h}_{\omega}(\boldsymbol{\alpha} \frown \gamma)\right) \cdot \omega$.

Case 2: $\gamma_{1} \leq \alpha_{n}$. Here we argue similarly as in the proof of part 4, case 2. However, the access to the i.h. is different. Clearly, $\kappa_{\alpha_{n} \cdot \log \left(\gamma_{i}\right)}^{\boldsymbol{\alpha}}=\mathrm{o}(\boldsymbol{\alpha}) \cdot \log \left(\gamma_{i}\right)$. For given $i$, let $\log \left(\log \left(\gamma_{i}\right)\right)={ }_{\mathrm{ANF}} \xi_{1}+\ldots+\xi_{s}$. In the case $\gamma_{i}=\alpha_{n}$ we have $\operatorname{dp}_{\boldsymbol{\alpha}}\left(\alpha_{n}^{2}\right)=\mathrm{o}(\boldsymbol{\alpha})$. Now assume that $\gamma_{i}<\alpha_{n}$. An application of part 1 of the i.h. to $\xi_{j}$ yields $\left(\kappa_{\xi_{j}}^{\boldsymbol{\alpha}}+\operatorname{dp}_{\boldsymbol{\alpha}}\left(\xi_{j}\right)\right) \cdot \omega<\mathrm{o}(\boldsymbol{\alpha})$ for $j=1, \ldots, s$. Therefore

$$
\left(\kappa_{\alpha_{n} \cdot \log \left(\gamma_{i}\right)}^{\boldsymbol{\alpha}}+\operatorname{dp}_{\boldsymbol{\alpha}}\left(\alpha_{n} \cdot \log \left(\gamma_{i}\right)\right)\right) \cdot \omega=\kappa_{\alpha_{n} \cdot \log \left(\gamma_{i}\right)}^{\boldsymbol{\alpha}} \cdot \omega=\mathrm{o}(\boldsymbol{\alpha}) \cdot \log \left(\gamma_{i}\right) \cdot \omega
$$

These considerations show that we obtain

$$
\left(\nu_{\gamma}^{\boldsymbol{\alpha}}+\kappa_{\varrho_{\gamma}^{\alpha_{n}}}^{\boldsymbol{\alpha}}+\operatorname{dp}_{\boldsymbol{\alpha}}\left(\varrho_{\gamma}^{\alpha_{n}}\right)\right) \cdot \omega=\nu_{\gamma}^{\boldsymbol{\alpha}} \cdot \omega=\mathrm{o}(\boldsymbol{\alpha}) \cdot \gamma \cdot \omega
$$

Case 3: Otherwise.

Subcase 3.1: $\varrho_{\gamma} \in \mathbb{E}^{>\alpha_{n}}$. Since $\gamma \notin \mathbb{E}^{>\alpha_{n}}$ we have $\log (\gamma)=\lambda+1$ where $\lambda \in \mathbb{E}^{>\alpha_{n}}$ and $\chi^{\alpha_{n}}(\lambda)=1$. Hence $\gamma=\lambda \cdot \omega$ and $\varrho_{\gamma}=\lambda$, for which part 4 of the i.h. yields

$$
\left(\kappa_{\lambda}^{\boldsymbol{\alpha}}+\operatorname{dp}_{\boldsymbol{\alpha}}(\lambda)\right) \cdot \omega=\operatorname{dp}_{\boldsymbol{\alpha}}(\lambda) \cdot \omega=\mathrm{o}\left(\mathrm{h}_{\omega}(\boldsymbol{\alpha} \frown \lambda)\right) \cdot \omega=\mathrm{o}(\boldsymbol{\alpha} \frown \gamma),
$$

implying the claim. 
Subcase 3.2: $\varrho_{\gamma} \notin \mathbb{E}^{>\alpha_{n}}$. Here we extend the argumentation from case 2, where the situation $\gamma_{i} \leq \alpha_{n}$ has been resolved. In the case $\gamma_{i} \in \mathbb{E}^{>\alpha_{n}}$ we have $\gamma_{i} \cdot \omega \leq \gamma$ and apply part 4 of the i.h. to $\gamma_{i}$ to obtain

$$
\left(\kappa_{\alpha_{n} \cdot \log \left(\gamma_{i}\right)}^{\boldsymbol{\alpha}}+\mathrm{dp}_{\boldsymbol{\alpha}}\left(\alpha_{n} \cdot \log \left(\gamma_{i}\right)\right)\right) \cdot \omega=\mathrm{dp}_{\boldsymbol{\alpha}}\left(\gamma_{i}\right) \cdot \omega=\mathrm{o}\left(\boldsymbol{\alpha}^{\frown} \gamma_{i} \cdot \omega\right) \leq \mathrm{o}\left(\boldsymbol{\alpha}^{\frown} \gamma\right) .
$$

We are left with the cases where $\gamma_{i} \in \mathbb{M}^{>\alpha_{n}}-\mathbb{E}$. Writing $\log \left(\log \left(\gamma_{i}\right)\right)={ }_{\mathrm{ANF}} \xi_{1}+\ldots+\xi_{s}$, which resides in $\left(\alpha_{n}, \log \left(\gamma_{i}\right)\right)$, we have $\operatorname{dp}_{\boldsymbol{\alpha}}\left(\alpha_{n} \cdot \log \left(\gamma_{i}\right)\right)=\sum_{j=1}^{s}\left(\kappa_{\xi_{j}}^{\boldsymbol{\alpha}}+\mathrm{dp}_{\boldsymbol{\alpha}}\left(\xi_{j}\right)\right)$, where for each $j$ part 1 of the i.h. applied to $\xi_{j}$ together with the monotonicity of $\kappa^{\alpha}$ on additive principal arguments yield

$$
\left(\kappa_{\xi_{j}}^{\boldsymbol{\alpha}}+\operatorname{dp}_{\boldsymbol{\alpha}}\left(\xi_{j}\right)\right) \cdot \omega=\kappa_{\xi_{j} \cdot \omega}^{\boldsymbol{\alpha}} \leq \kappa_{\alpha_{n} \cdot \log \left(\gamma_{i}\right)}^{\boldsymbol{\alpha}}<\nu_{\gamma}^{\boldsymbol{\alpha}},
$$

and we conclude as in case 2 .

Corollary 4.7 Let $\boldsymbol{\alpha} \in \mathrm{RS}$. We have

$$
\begin{aligned}
& \text { 1. } \kappa_{\gamma \cdot \omega}^{\boldsymbol{\alpha}}=\left(\kappa_{\gamma}^{\boldsymbol{\alpha}}+\operatorname{dp}_{\boldsymbol{\alpha}}(\gamma)\right) \cdot \omega \text { for } \gamma \in \mathbb{P} \text { such that } \gamma \cdot \omega \in \operatorname{dom}\left(\kappa^{\boldsymbol{\alpha}}\right) \text {. } \\
& \text { 2. } \nu_{\gamma \cdot \omega}^{\boldsymbol{\alpha}}=\left(\nu_{\gamma}^{\boldsymbol{\alpha}}+\kappa_{\varrho_{\gamma}^{\alpha_{n}}}^{\boldsymbol{\alpha}}+\operatorname{dp}_{\boldsymbol{\alpha}}\left(\varrho_{\gamma}^{\alpha_{n}}\right)\right) \cdot \omega \text { for } \gamma \in \mathbb{P} \text { such that } \gamma \cdot \omega \in \operatorname{dom}\left(\nu^{\boldsymbol{\alpha}}\right) \text {. }
\end{aligned}
$$

$\kappa^{\boldsymbol{\alpha}}$ and for $\boldsymbol{\alpha} \neq()$ also $\nu^{\boldsymbol{\alpha}}$ are strictly monotonically increasing and continuous.

Proof. Parts 1 and 2 follow from Definitions 3.14 and 4.1 using parts 1 and 5 of Lemma 4.6 respectively. In order to see general monotonicity and continuity we can build upon Corollary 4.2 The missing argument is as follows. For any $\beta \in \mathbb{P}$ in the respective domain and any $\gamma={ }_{\mathrm{ANF}} \gamma_{1}+\ldots+\gamma_{m}<\beta$ we have $\kappa_{\gamma}^{\boldsymbol{\alpha}}<\kappa_{\beta}^{\boldsymbol{\alpha}}$ using part 1 , and $\nu_{\gamma}^{\alpha}<\nu_{\beta}^{\boldsymbol{\alpha}}$ using part 2 , since $\gamma_{i} \cdot \omega \leq \beta$ for $i=1, \ldots, m$.

Theorem 4.8 Let $\boldsymbol{\alpha}=\left(\alpha_{1}, \ldots, \alpha_{n}\right) \in \mathrm{RS}, n \geq 0$, and set $\alpha_{0}:=1$. For $\beta \in \mathbb{P}$ let $\delta:=(1 / \bar{\beta}) \cdot \beta$, so that $\beta={ }_{\mathrm{NF}} \bar{\beta} \cdot \delta$ if $\beta \notin \mathbb{M}$.

1. For all $\beta \in \operatorname{dom}\left(\kappa^{\alpha}\right) \cap \mathbb{P}^{>\alpha_{n}}$ we have

$$
\kappa_{\beta}^{\alpha}=\kappa_{\bar{\beta}+1}^{\alpha} \cdot \delta
$$

2. For all $\beta \in \operatorname{dom}\left(\nu^{\alpha}\right) \cap \mathbb{P}^{>\alpha_{n}}$ (where $n>0$ ) we have

$$
\nu_{\beta}^{\alpha}=\nu_{\bar{\beta}+1}^{\alpha} \cdot \delta
$$

Hence, the definitions of $\kappa, \nu$, and dp given in [5] and here fully agree.

Proof. We rely on the monotonicity of o. Note that Corollary 4.7 has already shown the theorem for $\beta$ of the form $\gamma \cdot \omega$, i.e. successors of additive principal numbers. Let $\beta={ }_{\mathrm{MNF}} \beta_{1} \cdot \ldots \cdot \beta_{k} \in \mathbb{P}^{>\alpha_{n}}$.

Case 1: $k=1$. Then $\delta=\beta$, and setting $n_{0}:=n_{0}(\boldsymbol{\alpha} \frown \beta)$ and $\gamma:=\gamma(\boldsymbol{\alpha} \frown \beta)$ according to Definition 3.14, we have

$$
\kappa_{\beta}^{\boldsymbol{\alpha}}=\nu_{\beta}^{\boldsymbol{\alpha}}=\mathrm{o}(\boldsymbol{\alpha} \frown \beta)= \begin{cases}\beta & \text { if } n_{0}=0 \\ \mathrm{o}\left(\boldsymbol{\alpha}^{\prime}\right) \cdot \beta & \text { if } n_{0}>0,\end{cases}
$$

where $\boldsymbol{\alpha}^{\prime}:=\mathrm{h}_{\beta}\left(\boldsymbol{\alpha}_{\left.\right|_{n_{0}-1}} \frown \gamma\right)$, and parts 1 and 5 of Lemma 4.6 yield

$$
\kappa_{\bar{\beta}+1}^{\boldsymbol{\alpha}} \cdot \beta=\nu_{\bar{\beta}+1}^{\boldsymbol{\alpha}} \cdot \beta= \begin{cases}\mathrm{o}(\boldsymbol{\alpha}) \cdot \beta & \text { if } \bar{\beta}=\alpha_{n} \\ \mathrm{o}\left(\mathrm{h}_{\omega}\left(\boldsymbol{\alpha}^{-} \bar{\beta}\right)\right) \cdot \beta & \text { if } \bar{\beta}>\alpha_{n} .\end{cases}
$$

If $n_{0}=0$ the claim is immediate since $\mathrm{o}(\boldsymbol{\alpha}) \cdot \beta=\beta$ if $\bar{\beta}=\alpha_{n}$ and $1<\mathrm{o}\left(\mathrm{h}_{\omega}(\boldsymbol{\alpha} \frown \bar{\beta})\right)<\mathrm{o}(\boldsymbol{\alpha} \frown \beta)=\beta$ if $\bar{\beta}>\alpha_{n}$. Now assume that $n_{0}>0$.

Subcase 1.1: $\bar{\beta}=\alpha_{n}$. This implies $n_{0} \leq n$ and therefore $\boldsymbol{\alpha}^{\prime}<_{\operatorname{lex}} \boldsymbol{\alpha}<_{\operatorname{lex}} \boldsymbol{\alpha} \frown \beta$. By the monotonicity of o we obtain

$$
\mathrm{o}\left(\boldsymbol{\alpha}^{\prime}\right)<\mathrm{o}\left(\boldsymbol{\alpha}^{\frown \beta}\right)=\mathrm{o}\left(\boldsymbol{\alpha}^{\prime}\right) \cdot \beta
$$

which implies the claim since $\beta \in \mathbb{M}$. 
Subcase 1.2: $\bar{\beta}>\alpha_{n}$. This implies $\bar{\beta} \in \mathbb{E}^{>\alpha_{n}}$ and $\kappa_{\bar{\beta}+1}^{\boldsymbol{\alpha}} \cdot \beta=\nu_{\bar{\beta}+1}^{\boldsymbol{\alpha}} \cdot \beta=\mathrm{o}\left(\mathrm{h}_{\omega}(\boldsymbol{\alpha} \frown \bar{\beta})\right) \cdot \beta$, where $\mathrm{h}_{\omega}(\boldsymbol{\alpha} \frown \bar{\beta})<_{\text {lex }} \boldsymbol{\alpha} \frown \beta$.

1.2.1: $n_{0} \leq n$. Then we obtain $\boldsymbol{\alpha}^{\prime}<_{\text {lex }} \boldsymbol{\alpha}<_{\text {lex }} \mathrm{h}_{\omega}(\boldsymbol{\alpha} \frown \bar{\beta})<_{\text {lex }} \boldsymbol{\alpha} \frown \beta$ and hence

$$
\mathrm{o}\left(\boldsymbol{\alpha}^{\prime}\right)<\mathrm{o}\left(\mathrm{h}_{\omega}\left(\boldsymbol{\alpha}^{\frown} \bar{\beta}\right)\right)<\mathrm{o}\left(\boldsymbol{\alpha}^{\frown} \beta\right)=\mathrm{o}\left(\boldsymbol{\alpha}^{\prime}\right) \cdot \beta
$$

which implies the claim.

1.2.2: $n_{0}=n+1$. Then we have $\gamma \leq \bar{\beta}, \boldsymbol{\alpha}^{\prime}=\mathrm{h}_{\beta}(\boldsymbol{\alpha} \frown \gamma)$, and using Corollary 3.13] it follows that

$$
\kappa_{\beta}^{\boldsymbol{\alpha}}=\nu_{\beta}^{\boldsymbol{\alpha}}=\mathrm{o}\left(\boldsymbol{\alpha}^{\frown} \beta\right)=\mathrm{o}\left(\boldsymbol{\alpha}^{\prime}\right) \cdot \beta>\mathrm{o}\left(\mathrm{h}_{\omega}\left(\boldsymbol{\alpha}^{\frown} \bar{\beta}\right)\right) \geq \mathrm{o}\left(\mathrm{h}_{\beta}\left(\boldsymbol{\alpha}^{\frown} \gamma\right)\right)
$$

which again implies the claim.

Case 2: $k>1$. Then we have $\bar{\beta}={ }_{\mathrm{MNF}} \beta_{1} \cdot \ldots \cdot \beta_{k-1} \geq \alpha_{n}, \delta=\beta_{k}$, and set $\beta^{\prime}:=\left(1 / \beta_{1}\right) \cdot \beta$ and $\bar{\beta}^{\prime}:=\left(1 / \beta_{1}\right) \cdot \bar{\beta}$.

Subcase 2.1: $\beta_{1}=\alpha_{n}$. Using Lemma 4.6 we obtain

$$
\kappa_{\bar{\beta}+1}^{\boldsymbol{\alpha}} \cdot \delta=\mathrm{o}(\boldsymbol{\alpha}) \cdot \beta^{\prime}=\kappa_{\beta}^{\boldsymbol{\alpha}}
$$

and

$$
\nu_{\bar{\beta}+1}^{\boldsymbol{\alpha}} \cdot \delta=\mathrm{o}(\boldsymbol{\alpha}) \cdot \beta=\nu_{\beta}^{\boldsymbol{\alpha}} .
$$

Subcase 2.2: $\beta_{1}>\alpha_{n}$. Then we have $\kappa_{\beta}^{\boldsymbol{\alpha}}=\nu_{\beta}^{\boldsymbol{\alpha}}$.

2.2.1: $\bar{\beta} \notin \mathbb{E}^{>\alpha_{n}}$. Then by the involved definitions

$$
\kappa_{\bar{\beta}+1}^{\boldsymbol{\alpha}} \cdot \delta=\nu_{\bar{\beta}+1}^{\boldsymbol{\alpha}} \cdot \delta=\mathrm{o}\left(\boldsymbol{\alpha}^{\frown \bar{\beta}}\right) \cdot \delta=\nu_{\beta}^{\boldsymbol{\alpha}}=\kappa_{\beta}^{\boldsymbol{\alpha}} .
$$

2.2.2: $\bar{\beta} \in \mathbb{E}^{>\alpha_{n}}$. This implies $k=2, \delta=\beta_{2}$, and we see that

$$
\kappa_{\bar{\beta}+1}^{\boldsymbol{\alpha}} \cdot \delta=\nu_{\bar{\beta}+1}^{\boldsymbol{\alpha}} \cdot \delta=\mathrm{o}\left(\mathrm{h}_{\omega}\left(\boldsymbol{\alpha}^{\frown} \bar{\beta}\right)\right) \cdot \delta .
$$

In the case $\delta>\mu_{\beta_{1}}$ we have $\mathrm{h}_{\delta}(\boldsymbol{\alpha} \frown \bar{\beta})=\boldsymbol{\alpha} \frown \bar{\beta}$ and hence obtain uniformly

$$
\kappa_{\beta}^{\boldsymbol{\alpha}}=\nu_{\beta}^{\boldsymbol{\alpha}}=\mathrm{o}\left(\mathrm{h}_{\delta}(\boldsymbol{\alpha} \frown \bar{\beta})\right) \cdot \delta .
$$

By Corollary 3.12 we have $\mathrm{h}_{\delta}(\boldsymbol{\alpha} \frown \bar{\beta}) \leq_{\text {lex }} \mathrm{h}_{\omega}(\boldsymbol{\alpha} \frown \bar{\beta})$, hence

$$
\mathrm{o}\left(\mathrm{h}_{\delta}(\boldsymbol{\alpha} \frown \bar{\beta})\right) \leq \mathrm{o}\left(\mathrm{h}_{\omega}(\boldsymbol{\alpha} \frown \bar{\beta})\right)<\mathrm{o}(\boldsymbol{\alpha} \frown \beta)=\mathrm{o}\left(\mathrm{h}_{\delta}(\boldsymbol{\alpha} \frown \bar{\beta})\right) \cdot \delta
$$

which implies the claim since $\delta \in \mathbb{M}$.

Lemma 4.9 Let $\boldsymbol{\alpha}=\left(\alpha_{1}, \ldots, \alpha_{n}\right) \in \mathrm{RS}, n>0$.

1. For all $\beta$ such that $\boldsymbol{\alpha} \frown \beta \in \mathrm{TS}$ we have

$$
\mathrm{o}(\boldsymbol{\alpha} \frown \beta)<\mathrm{o}(\boldsymbol{\alpha}) \cdot \widehat{\alpha_{n}}
$$

2. For all $\gamma$ such that $\boldsymbol{\alpha} \frown \gamma \in \mathrm{RS}$ we have

$$
\mathrm{o}\left(\mathrm{h}_{\omega}(\boldsymbol{\alpha} \frown \gamma)\right)<\mathrm{o}(\boldsymbol{\alpha} \frown \gamma) \cdot \widehat{\gamma}
$$

Proof. We prove the lemma by simultaneous induction on $\operatorname{lSeq}(\boldsymbol{\alpha} \frown \beta)$ and $\operatorname{lSeq}\left(\mathrm{h}_{\omega}(\boldsymbol{\alpha} \frown \gamma)\right)$, respectively.

Ad 1. Let $\beta={ }_{\mathrm{MNF}} \beta_{1} \cdot \ldots \cdot \beta_{k}$ and $\beta^{\prime}:=\left(1 / \beta_{1}\right) \cdot \beta$. Note that $\beta \leq \mu_{\alpha_{n}}<\widehat{\alpha}$.

Case 1: $\beta_{1} \leq \alpha_{n}$. Immediate, since $\mathrm{o}(\boldsymbol{\alpha} \frown \beta)=\mathrm{o}(\boldsymbol{\alpha}) \cdot \beta$.

Case 2: $k>1$ where $\beta_{1} \in \mathbb{E}^{>\alpha_{n}}$ and $\beta_{2} \leq \mu_{\beta_{1}}$. Then we have o $(\boldsymbol{\alpha} \frown \beta)=\mathrm{o}\left(\mathrm{h}_{\beta_{2}}\left(\boldsymbol{\alpha} \frown \beta_{1}\right)\right) \cdot \beta^{\prime}$ and apply the i.h. to $\boldsymbol{\alpha} \frown \beta_{1}$, which clearly satisfies $\operatorname{lSeq}\left(\boldsymbol{\alpha}^{\frown} \beta_{1}\right)<_{\text {lex }} \operatorname{lSeq}(\boldsymbol{\alpha} \frown \beta)$. By Corollaries 3.13 and 3.21 we have $\mathrm{o}\left(\mathrm{h}_{\beta_{2}}\left(\boldsymbol{\alpha} \frown \beta_{1}\right)\right) \leq \mathrm{o}\left(\mathrm{h}_{\omega}\left(\boldsymbol{\alpha} \frown \beta_{1}\right)\right)$, and by the i.h., parts $1\left(\right.$ for $\left.\boldsymbol{\alpha} \frown \beta_{1}\right)$ and $2\left(\right.$ for $\left.\mathrm{h}_{\omega}\left(\boldsymbol{\alpha} \frown \beta_{1}\right)\right)$ we obtain

$$
\mathrm{o}\left(\mathrm{h}_{\omega}\left(\boldsymbol{\alpha} \frown \beta_{1}\right)\right)<\mathrm{o}\left(\boldsymbol{\alpha} \frown \beta_{1}\right) \cdot \widehat{\beta_{1}}<\mathrm{o}(\boldsymbol{\alpha}) \cdot \widehat{\alpha_{n}},
$$


where we have used that $\widehat{\beta_{1}} \leq \widehat{\alpha_{n}}$ according to Lemma 3.17 of [5]. This implies the desired inequality.

Case 3: Otherwise. Let $n_{0}:=n_{0}\left(\boldsymbol{\alpha}^{\frown} \beta\right)$ and $\gamma:=\gamma\left(\boldsymbol{\alpha}^{\frown} \beta\right)$ according to Definition 3.14,

Subcase 3.1: $n_{0}=0$. Immediate.

Subcase 3.2: $n_{0}>0$. By definition we have $o\left(\boldsymbol{\alpha}^{\frown} \beta\right)=\mathrm{o}\left(\mathrm{h}_{\beta_{1}}\left(\boldsymbol{\alpha}_{\left\lceil_{n_{0}-1}\right.} \frown \gamma\right)\right) \cdot \beta$.

3.2.1: $n_{0} \leq n$. The monotonicity of o then yields o $(\boldsymbol{\alpha} \frown \beta) \leq \mathrm{o}(\boldsymbol{\alpha}) \cdot \beta<\mathrm{o}(\boldsymbol{\alpha}) \cdot \widehat{\alpha_{n}}$.

3.2.2: $n_{0}=n+1$. Then $\gamma$ is the immediate predecessor of $\beta$ in $\operatorname{ts}^{\alpha_{n}}(\beta)$. We apply the i.h. for parts 1 (to $\boldsymbol{\alpha}^{-} \gamma$ ) and $2\left(\right.$ to $\left.\mathrm{h}_{\omega}\left(\boldsymbol{\alpha}^{\frown} \gamma\right)\right)$ and argue as in Case 2 to see that $\mathrm{o}(\boldsymbol{\alpha} \frown \beta)=\mathrm{o}\left(\mathrm{h}_{\beta_{1}}\left(\boldsymbol{\alpha}^{\frown} \gamma\right)\right) \cdot \beta<\mathrm{o}(\boldsymbol{\alpha}) \cdot \widehat{\alpha_{n}}$.

Ad 2. We have $\mathrm{h}_{\omega}\left(\boldsymbol{\alpha}^{\frown} \gamma\right)=\boldsymbol{\alpha}^{\frown} \operatorname{sk}_{\omega}(\gamma)$, and setting $\operatorname{sk}_{\omega}(\gamma)=:\left(\delta_{1}, \ldots, \delta_{l+1}\right)$ we obtain a strictly increasing sequence of $\mathbb{E}$-numbers $\boldsymbol{\delta}:=\left(\delta_{1}, \ldots, \delta_{l}\right)=\left(\gamma, \mu_{\gamma}, \mu_{\mu_{\gamma}}, \ldots\right)$ such that $\delta_{l+1}=\mu_{\delta_{l}} \notin \mathbb{E}^{>\delta_{l}}$. For $i=1, \ldots, l$ we have

$$
\begin{gathered}
\mathrm{h}_{\omega}\left(\boldsymbol{\alpha} \frown \boldsymbol{\delta}_{\left.\right|_{i}}\right)=\boldsymbol{\alpha} \frown \boldsymbol{\delta}^{\frown} \delta_{l+1}, \\
\operatorname{lSeq}\left(\boldsymbol{\alpha} \frown \boldsymbol{\delta}_{\left.\right|_{i}}\right) \leq_{\operatorname{lex}} \operatorname{lSeq}\left(\mathrm{h}_{\omega}(\boldsymbol{\alpha} \frown \gamma)\right),
\end{gathered}
$$

and the i.h., part 1 (up to $\mathrm{h}_{\omega}(\boldsymbol{\alpha} \frown \gamma)$ ), yields

$$
\mathrm{o}\left(\boldsymbol{\alpha}^{\frown} \boldsymbol{\delta}_{\uparrow_{i+1}}\right)<\mathrm{o}\left(\boldsymbol{\alpha} \frown \boldsymbol{\delta}_{\uparrow_{i}}\right) \cdot \widehat{\delta_{i}} .
$$

Appealing to Lemma 3.17 of [5] we obtain

$$
\widehat{\delta_{l}} \leq \widehat{\delta_{l-1}} \leq \ldots \leq \widehat{\delta_{1}}=\widehat{\gamma},
$$

and finally conclude that $\mathrm{o}\left(\mathrm{h}_{\omega}\left(\boldsymbol{\alpha}^{\frown} \gamma\right)\right)<\mathrm{o}\left(\boldsymbol{\alpha}^{\frown} \gamma\right) \cdot \widehat{\gamma}$.

Corollary 4.10 For all $\boldsymbol{\alpha}^{\frown} \gamma \in \mathrm{RS}$ the ordinal $\mathrm{o}\left(\boldsymbol{\alpha}^{\frown} \gamma\right) \cdot \widehat{\gamma}$ is a strict upper bound of

$$
\operatorname{Im}\left(\kappa^{\boldsymbol{\alpha}^{-} \gamma}\right), \operatorname{Im}\left(\nu^{\boldsymbol{\alpha}^{-} \gamma}\right), \operatorname{dp}_{\boldsymbol{\alpha}}(\gamma) \text {, and } \nu_{\mu_{\gamma}}^{\boldsymbol{\alpha} \gamma}+\kappa_{\lambda_{\gamma}}^{\boldsymbol{\alpha}^{-\gamma}}+\operatorname{dp}_{\boldsymbol{\alpha} \frown \gamma}\left(\lambda_{\gamma}\right) \text {. }
$$

Proof. This directly follows from Lemmas 4.6 and 4.9 ,

\section{$5 \quad$ Revisiting tracking chains}

\subsection{Preliminary remarks}

Our preparations in the previous sections are almost sufficient to demonstrate that the characterization of $\mathcal{C}_{2}$ provided in Section 7 of [5] is elementary recursive. We first provide a brief argumentation based on the previous sections showing that the structure $\mathcal{C}_{2}$ is elementary recursive. In the following subsections we will elaborate on the characterization of $\leq_{1}$ and $\leq_{2}$ within $\mathcal{C}_{2}$, further illuminating the structure.

In Section 5 of [5] the termination of the process of maximal extension (see Definition 5.2 of [5]) is seen when applying the $\mathrm{l}^{\tau}$-measure from the second step on, as clause 2.3.1 of Def. 5.2 in [5] can only be applied at the beginning of the process of maximal extension. Lemma 5.4, part a), of [5] is not needed in full generality, the $1^{\tau}$-measure suffices, cf. Lemma 5.5 of [5]. The proof of Lemma 5.12 of [5], parts a), b), and c), actually proceeds by induction on the number of 1-step extensions, an "induction on $\operatorname{cs}^{\prime}(\boldsymbol{\alpha})$ along $<_{\text {lex }}$ " is not needed.

In Section 6 of [5] the proof of Lemma 6.2 actually proceeds by induction on the length of the additive decomposition of $\alpha$. Definition 6.1 of [5], which assigns to each $\alpha<1^{\infty}$ its unique tracking chain tc $(\alpha)$, involves the evaluation function o (in the guise of $\tilde{r}$, cf. Definition 5.9 of [5]), which we have shown to be elementary recursive.

\subsection{Pre-closed and spanning sets of tracking chains}

For the formal definition of tracking chains recall Definition 5.1 of [5]. We will rely on its detailed terminology, including the notation $\boldsymbol{\alpha} \subseteq \boldsymbol{\beta}$ when $\boldsymbol{\alpha}$ is an initial chain of $\boldsymbol{\beta}$.

The following definitions of pre-closed and spanning sets of tracking chains provide a generalization of the notion of maximal extension, denoted by me, cf. Definition 5.2 of [5]. 
Definition 5.1 (Pre-closedness) Let $M \subseteq_{\text {fin }}$ TC. $M$ is pre-closed if and only if $M$

1. is closed under initial chains: if $\boldsymbol{\alpha} \in M$ and $(i, j) \in \operatorname{dom}(\boldsymbol{\alpha})$ then $\boldsymbol{\alpha}_{\uparrow_{(i, j)}} \in M$,

2. is $\nu$-index closed: if $\boldsymbol{\alpha} \in M, m_{n}>1, \alpha_{n, m_{n}}={ }_{\mathrm{ANF}} \xi_{1}+\ldots+\xi_{k}$ then $\boldsymbol{\alpha}\left[\mu_{\tau^{\prime}}\right], \boldsymbol{\alpha}\left[\xi_{1}+\ldots+\xi_{l}\right] \in M$ for $1 \leq l \leq k$,

3. unfolds minor $\leq_{2}$-components: if $\boldsymbol{\alpha} \in M, m_{n}>1$, and $\tau<\mu_{\tau^{\prime}}$ then:

3.1. $\boldsymbol{\alpha}_{\uparrow_{n-1}}-\left(\alpha_{n, 1}, \ldots, \alpha_{n, m_{n}}, \mu_{\tau}\right) \in M$ in the case $\tau \in \mathbb{E}^{>\tau^{\prime}}$, and

3.2. otherwise $\boldsymbol{\alpha} \frown\left(\varrho_{\tau}^{\tau^{\prime}}\right) \in M$, provided that $\varrho_{\tau}^{\tau^{\prime}}>0$,

4. is $\kappa$-index closed: if $\boldsymbol{\alpha} \in M, m_{n}=1$, and $\alpha_{n, 1}={ }_{\mathrm{ANF}} \xi_{1}+\ldots+\xi_{k}$, then:

4.1. if $m_{n-1}>1$ and $\xi_{1}=\tau_{n-1, m_{n-1}} \in \mathbb{E}^{>\tau_{n-1, m_{n-1}-1}}$ then $\boldsymbol{\alpha}_{\uparrow_{n-2}}-\left(\alpha_{n-1,1}, \ldots, \alpha_{n-1, m_{n-1}}, \mu_{\xi_{1}}\right) \in M$, else $\boldsymbol{\alpha}_{\uparrow_{n-1}} \frown\left(\xi_{1}\right) \in M$, and

4.2. $\boldsymbol{\alpha}_{\left.\right|_{n-1}} \frown\left(\xi_{1}+\ldots+\xi_{l}\right) \in M$ for $l=2, \ldots, k$,

5. maximizes me- $\mu$-chains: if $\boldsymbol{\alpha} \in M, m_{n} \geq 1$, and $\tau \in \mathbb{E}^{>\tau^{\prime}}$, then:

5.1. if $m_{n}=1$ then $\boldsymbol{\alpha}_{\left.\right|_{n-1}} \frown\left(\alpha_{n, 1}, \mu_{\tau}\right) \in M$, and

5.2. if $m_{n}>1$ and $\tau=\mu_{\tau^{\prime}}=\lambda_{\tau^{\prime}}$ then $\boldsymbol{\alpha}_{\left.\right|_{n-1}} \frown\left(\alpha_{n, 1} \ldots, \alpha_{n, m_{n}}, \mu_{\tau}\right) \in M$.

Remark. Pre-closure of some $M \subseteq_{\text {fin }}$ TC is obtained by closing under clauses $1-5$ in this order once, hence finite: in clause 5 note that $\mu$-chains are finite since the ht-measure of terms strictly decreases with each application of $\mu$. Note further that intermediate indices are of the form $\lambda_{\tau^{\prime}}$, whence we have a decreasing l-measure according to inequality 6 in the remark following Definition 4.3 .

Definition 5.2 (Spanning sets of tracking chains) $M \subseteq_{\text {fin }}$ TC is spanning if and only if it is pre-closed and closed under

6. unfolding of $\leq_{1}$-components: for $\boldsymbol{\alpha} \in M$, if $m_{n}=1$ and $\tau \notin \mathbb{E}^{\geq \tau^{\prime}}$ (i.e. $\tau=\tau_{n, m_{n}} \notin \mathbb{E}_{1}, \tau^{\prime}=\tau_{n}^{\star}$ ), let

$$
\log \left(\left(1 / \tau^{\prime}\right) \cdot \tau\right)=_{\mathrm{ANF}} \xi_{1}+\ldots+\xi_{k},
$$

if otherwise $m_{n}>1$ and $\tau=\mu_{\tau^{\prime}}$ such that $\tau<\lambda_{\tau^{\prime}}$ in the case $\tau \in \mathbb{E}^{>\tau^{\prime}}$, let

$$
\lambda_{\tau^{\prime}}={ }_{\mathrm{ANF}} \xi_{1}+\ldots+\xi_{k} .
$$

Set $\xi:=\xi_{1}+\ldots+\xi_{k}$, unless $\xi>0$ and $\boldsymbol{\alpha} \frown\left(\xi_{1}+\ldots+\xi_{k}\right) \notin \mathrm{TC}, 3$ in which case we set $\xi:=\xi_{1}+\ldots+\xi_{k-1}$. Suppose that $\xi>0$. Let $\boldsymbol{\alpha}^{+}$denote the vector $\{\boldsymbol{\alpha}-(\xi)\}$ if this is a tracking chain, or otherwise the vector $\boldsymbol{\alpha}_{\left.\right|_{n-1}} \frown\left(\alpha_{n, 1}, \ldots, \alpha_{n, m_{n}}, \mu_{\tau_{n, m_{n}}}\right)$. 4 Then the closure of $\left\{\boldsymbol{\alpha}^{+}\right\}$under clauses 4 and 5 is contained in $M$.

Remark. Closure of some $M \subseteq_{\text {fin }}$ TC under clauses $1-6$ is a finite process since pre-closure is finite and since the $\kappa$-indices added in clause 6 strictly decrease in l-measure.

Semantically, the above notion of spanning sets of tracking chains and closure under clauses $1-6$ leaves some redundancy in the form that certain $\kappa$-indices could be omitted. This will be adressed elsewhere, since the current formulation is advantageous for technical reasons.

Definition 5.3 (Relativization) Let $\boldsymbol{\alpha} \in \mathrm{TC} \cup\{()\}$ and $M \subseteq_{\mathrm{fin}} \mathrm{TC}$ be a set of tracking chains that properly extend $\boldsymbol{\alpha} . M$ is pre-closed above $\boldsymbol{\alpha}$ if and only if it is pre-closed with the modification that clauses 1 - 5 only apply when the respective resulting tracking chains $\boldsymbol{\beta}$ properly extend $\boldsymbol{\alpha} . M$ is weakly spanning above $\boldsymbol{\alpha}$ if and only if $M$ is pre-closed above $\boldsymbol{\alpha}$ and closed under clause 6 .

Lemma 5.4 If $M$ is spanning (weakly spanning above some $\boldsymbol{\alpha}$ ), then it is closed under me (closed under me for proper extensions of $\boldsymbol{\alpha}$ ).

Proof. This follows directly from the definitions involved.

\footnotetext{
${ }^{3}$ This is the case if clause 6 of Def. 5.1 of [5] does not hold.

${ }^{4}$ This case distinction, due to clause 5 of Def. 5.1 of [5], is missing in [10].
} 


\subsection{Characterizing $\leq_{1}$ and $\leq_{2}$ in $\mathcal{C}_{2}$}

The purpose of this section is to provide a detailed picture of the restriction of $\mathcal{R}_{2}$ to $1^{\infty}$ on the basis of the results of [5] and to conclude with the extraction of an elementary recursive arithmetical characterization of this structure given in terms of tracking chains which we will refer to as $\mathcal{C}_{2}$.

We begin with a few observations that follow from the results in Section 7 of [5] and explain the concept of tracking chains. The evaluations of all initial chains of some tracking chain $\boldsymbol{\alpha} \in \mathrm{TC}$ form a $<_{1}$-chain. Evaluations of initial chains $\boldsymbol{\alpha}_{i, j}$ where $(i, j) \in \operatorname{dom}(\boldsymbol{\alpha})$ and $j=2, \ldots, m_{i}$ with fixed index $i$ form $<{ }_{2}$-chains. Recall that indices $\alpha_{i, j}$ are $\kappa$-indices for $j=1$ and $\nu$-indices otherwise, cf. Definitions 5.1 and 5.9 of [5].

According to Theorem 7.9 of [5], an ordinal $\alpha<1^{\infty}$ is $\leq_{1}$-minimal if and only if its tracking chain consists of a single $\kappa$-index, i.e. if its tracking chain $\boldsymbol{\alpha}$ satisfies $\left(n, m_{n}\right)=(1,1)$. Clearly, the least $\leq_{1}$-predecessor of any ordinal $\alpha<1^{\infty}$ with tracking chain $\boldsymbol{\alpha}$ is $\mathrm{o}\left(\boldsymbol{\alpha}_{\uparrow_{1,1}}\right)=\kappa_{\alpha_{1,1}}$. According to Corollary 7.11 of [5] the ordinal $1^{\infty}$ is $\leq_{1}$-minimal. An ordinal $\alpha>0$ has a non-trivial $\leq_{1}$-reach if and only if $\tau_{n, 1}>\tau_{n}^{\star}$, hence in particular when $m_{n}>1$, cf. condition 2 in Definition 5.1 of [ $[5]$.

We now turn to a characterization of the greatest immediate $\leq_{1}$-successor, gs $(\alpha)$, of an ordinal $\alpha<1^{\infty}$ with tracking chain $\boldsymbol{\alpha}$. Recall the notations $\rho_{i}$ and $\boldsymbol{\alpha}[\xi]$ from Definition 5.1 of [5]. The largest $\alpha-\leq_{1}$-minimal ordinal is the root of the $\lambda$ th $\alpha$ - $\leq_{1}$-component for $\lambda:=\rho_{n}-1$. Therefore, if $\alpha$ has a non-trivial $\leq_{1}$-reach, its greatest immediate $\leq_{1}$-successor gs $(\alpha)$ has the tracking chain $\boldsymbol{\alpha}-(\lambda)$, unless we either have $\tau_{n, m_{n}}<\mu_{\tau_{n}^{\prime}} \& \chi^{\tau_{n}^{\prime}}\left(\tau_{n, m_{n}}\right)=0$, where tc $(\operatorname{gs}(\alpha))=\boldsymbol{\alpha}\left[\alpha_{n, m_{n}}+1\right]$, or $\boldsymbol{\alpha}^{-}(\lambda)$ is in conflict with either condition 5 of Definition 5.1 of [5], in which case we have tc(gs $(\alpha))=\boldsymbol{\alpha}_{\uparrow_{n-1}} \frown\left(\alpha_{n, 1}, \ldots, \alpha_{n, m_{n}}, 1\right)$, or condition 6 of Definition 5.1 of [5], in which case we have $\operatorname{tc}(\operatorname{gs}(\alpha))=\boldsymbol{\alpha}_{i, j+1}\left[\alpha_{i, j+1}+1\right]$. 5 In case $\alpha$ does not have any $<_{1}$-successor, we set $\operatorname{gs}(\alpha):=\alpha$.

$\alpha$ is $\leq_{2}$-minimal if and only if for its tracking chain $\boldsymbol{\alpha}$ we have $m_{n} \leq 2$ and $\tau_{n}^{\star}=1$, and $\alpha$ has a non-trivial $\leq_{2}$-reach if and only if $m_{n}>1$ and $\tau_{n, m_{n}}>1$. Note that any $\alpha \in$ Ord with a non-trivial $\leq_{2}$-reach is the proper supremum of its $<_{1}$-predecessors, hence $1^{\infty}$ does not possess any $<_{2}$-successor. Iterated closure under the relativized notation system $\mathrm{T}^{\tau}$ for $\tau=1^{\infty},\left(1^{\infty}\right)^{\infty}, \ldots$ results in the infinite $<{ }_{2}$-chain through Ord. Its $<1_{1}$-root is $1^{\infty}$, the root of the "master main line" of $\mathcal{R}_{2}$, outside the core of $\mathcal{R}_{2}$, i.e. $1^{\infty}$.

According to part (a) of Theorem 7.9 of [5] $\alpha$ has a greatest $<_{1}$-predecessor if and only if it is not $\leq_{1}$-minimal and has a trivial $\leq_{2}$-reach (i.e. does not have any $<_{2}$-successor). This is the case if and only if either $m_{n}=1$ and $n>1$, where we have $\operatorname{pred}_{1}(\alpha)=\mathrm{o}_{n-1, m_{n-1}}(\boldsymbol{\alpha})$, or $m_{n}>1$ and $\tau_{n, m_{n}}=1$. In this latter case $\alpha_{n, m_{n}}$ is of a form $\xi+1$ for some $\xi \geq 0$, and using again the notation from Definition 5.1 of [5] we have $\operatorname{pred}_{1}(\alpha)=o(\boldsymbol{\alpha}[\xi])$ if $\chi^{\tau_{n, m_{n}-1}}(\xi)=0$, whereas $\operatorname{pred}_{1}(\alpha)=\mathrm{o}(\operatorname{me}(\boldsymbol{\alpha}[\xi]))$ in the case $\chi^{\tau_{n, m_{n}-1}}(\xi)=1$.

Recall Definition 7.12 of [5], defining for $\boldsymbol{\alpha} \in$ TC the notation $\boldsymbol{\alpha}^{\star}$ and the index pair gbo $(\boldsymbol{\alpha})=:\left(n_{0}, m_{0}\right)$, which according to Corollary 7.13 of [5] enables us to express the $\leq_{1}$-reach $\operatorname{lh}(\alpha)$ of $\alpha:=\mathrm{o}(\boldsymbol{\alpha})$, cf. Definition 7.7 of [5], by

$$
\ln (\alpha)=\mathrm{o}\left(\operatorname{me}\left(\boldsymbol{\beta}^{\star}\right)\right)
$$

where $\boldsymbol{\beta}:=\boldsymbol{\alpha}_{\uparrow_{n_{0}, m_{0}}}$, which in the case $m_{0}=1$ is equal to o(me $\left.(\boldsymbol{\beta})\right)=\mathrm{o}_{n_{0}, 1}(\boldsymbol{\alpha})+\mathrm{dp}_{\tilde{\tau}_{n_{0}, 0}}\left(\tau_{n_{0}, 1}\right)$ and in the case $m_{0}>1$ equal to o $\left(\operatorname{me}\left(\boldsymbol{\beta}\left[\mu_{\tau_{n_{0}, m_{0}-1}}\right]\right)\right)$. Note that if $\operatorname{cml}\left(\boldsymbol{\alpha}^{\star}\right)$ does not exist we have

$$
\ln (\alpha)=\mathrm{o}\left(\operatorname{me}\left(\boldsymbol{\alpha}^{\star}\right)\right)
$$

and the tracking chain $\boldsymbol{\beta}$ of any ordinal $\beta$ such that $\mathrm{o}\left(\boldsymbol{\alpha}^{\star}\right) \leq_{1} \beta$ is then an extension of $\boldsymbol{\alpha}, \boldsymbol{\alpha} \subseteq \boldsymbol{\beta}$, as will follow from Lemma 5.11 .

The relation $\leq_{1}$ can be characterized by

$$
\alpha \leq_{1} \beta \quad \Leftrightarrow \quad \alpha \leq \beta \leq \operatorname{lh}(\alpha),
$$

showing that $\leq_{1}$ is a forest contained in $\leq$, which respects the ordering $\leq$, i.e. if $\alpha \leq \beta \leq \gamma$ and $\alpha \leq_{1} \gamma$ then $\alpha \leq_{1} \beta$. On the basis of Lemma 5.4, Equation 7 has the following

Corollary 5.5 Let $M \subseteq_{\text {fin }}$ TC be spanning (weakly spanning above some $\boldsymbol{\alpha} \in \mathrm{TC}$ ) and $\boldsymbol{\beta} \in M, \boldsymbol{\beta}:=\mathrm{o}(\boldsymbol{\beta})$. Then

$$
\operatorname{tc}(\operatorname{lh}(\beta)) \in M,
$$

provided that $\mathrm{o}\left(\boldsymbol{\beta}_{\Gamma_{\mathrm{gbo}(\boldsymbol{\beta})}}\right)$ is a proper extension of $\boldsymbol{\alpha}$ in the case that $M$ is weakly spanning above $\boldsymbol{\alpha}$.

\footnotetext{
${ }^{5}$ This condition is missing in [10].
} 
We now recall how to retrieve the greatest $<_{2}$-predecessor of an ordinal below $1^{\infty}$, if it exists, and the iteration of this procedure to obtain the maximum chain of $<_{2}$-predecessors. Recall Definition 5.3 and Lemma 5.10 of [5]. Using the following proposition we can prove two other useful characterizations of the relationship $\alpha \leq_{2} \beta$.

Proposition 5.6 Let $\alpha<1^{\infty}$ with $\operatorname{tc}(\alpha)=$ : $\boldsymbol{\alpha}$. We define a sequence $\boldsymbol{\sigma} \in \mathrm{RS}$ as follows.

1. If $m_{n} \leq 2$ and $\tau_{n}^{\star}=1$, whence $\alpha$ is $\leq_{2}$-minimal according to Theorem 7.9 of [5], set $\boldsymbol{\sigma}:=()$. Otherwise,

2. if $m_{n}>2$, whence $\operatorname{pred}_{2}(\alpha)=\mathrm{o}_{n, m_{n}-1}(\boldsymbol{\alpha})$ with base $\tau_{n, m_{n}-2}$ according to Theorem 7.9 of [5], we set $\boldsymbol{\sigma}:=\operatorname{cs}\left(\boldsymbol{\alpha}_{\uparrow_{n, m_{n}-2}}\right)$,

3. and if $m_{n} \leq 2$ and $\tau_{n}^{\star}>1$, whence $\operatorname{pred}_{2}(\alpha)=\mathrm{o}_{i, j+1}(\boldsymbol{\alpha})$ with base $\tau_{i, j}$ where $(i, j):=n^{\star}$, again according to Theorem 7.9 of [5], we set $\boldsymbol{\sigma}:=\operatorname{cs}\left(\boldsymbol{\alpha}_{\uparrow_{i, j}}\right)$.

Each $\sigma_{i}$ is then of a form $\tau_{k, l}$ where $1 \leq l<m_{k}, 1 \leq k \leq n$. The corresponding $<_{2}$-predecessor of $\alpha$ is $\mathrm{o}_{k, l+1}(\boldsymbol{\alpha})=: \beta_{i}$. We obtain sequences $\boldsymbol{\sigma}=\left(\sigma_{1}, \ldots, \sigma_{r}\right)$ and $\boldsymbol{\beta}=\left(\beta_{1}, \ldots, \beta_{r}\right)$ with $\beta_{1}<_{2} \ldots<_{2} \beta_{r}<_{2} \alpha$, where $r=0$ if $\alpha$ is $\leq_{2}$-minimal, so that $\operatorname{Pred}_{2}(\alpha)=\left\{\beta_{1}, \ldots, \beta_{r}\right\}$ and hence $\beta<_{2} \alpha$ if and only if $\beta \in \operatorname{Pred}_{2}(\alpha)$, displaying that $\leq_{2}$ is a forest contained in $\leq_{1}$.

Lemma 5.7 Let $\alpha, \beta<1^{\infty}$ with tracking chains $\operatorname{tc}(\alpha)=\boldsymbol{\alpha}=\left(\boldsymbol{\alpha}_{1}, \ldots, \boldsymbol{\alpha}_{n}\right), \boldsymbol{\alpha}_{i}=\left(\alpha_{i, 1}, \ldots, \alpha_{i, m_{i}}\right), 1 \leq i \leq n$, and $\operatorname{tc}(\beta)=\boldsymbol{\beta}=\left(\boldsymbol{\beta}_{1}, \ldots, \boldsymbol{\beta}_{l}\right), \boldsymbol{\beta}_{i}=\left(\beta_{i, 1}, \ldots, \beta_{i, k_{i}}\right), 1 \leq i \leq l$. Assume further that $\boldsymbol{\alpha} \subseteq \boldsymbol{\beta}$ with associated chain $\tau$ and that $m_{n}>1$. Set $\tau:=\tau_{n, m_{n}-1}$. The following are equivalent:

1. $\alpha \leq_{2} \beta$

2. $\tau \leq \tau_{j, 1}$ for $j=n+1, \ldots, l$

3. $\tilde{\tau} \mid \beta$.

Proof. Note that $\boldsymbol{\alpha} \subseteq \boldsymbol{\beta}$ is a necessary condition for $\alpha \leq_{2} \beta$, cf. Proposition 5.6 .

$\mathbf{1} \Rightarrow \mathbf{2}$ : Iterating the operation $(\cdot)^{\prime}$ (which for $\tau_{j, 1}$ is $\tau_{j, 1}^{\prime}=\tau_{j}^{\star}$ ) then reaches $\tau$. This implies $\tau_{j, 1} \geq \tau$ for $j=n+1, \ldots, l$.

$\mathbf{2} \Rightarrow \mathbf{1}$ : Iterating the procedure to find the greatest $\leq_{2}$-predecessor, cf. Proposition 5.6, from $\beta$ downward satisfies $\tau_{j}^{\star} \geq \tau$ at each $j \in(n, l]$, where we therefore have $\left(n, m_{n}-1\right) \leq_{\text {lex }} j^{\star}$.

$\mathbf{2} \Rightarrow \mathbf{3}$ : Note that according to Lemma $5.10(\mathrm{c})$ of [5] we have

$$
\operatorname{ts}(\tilde{\tau})=\operatorname{cs}\left(\boldsymbol{\alpha}_{\uparrow_{n, m_{n}-1}}\right) .
$$

We argue by induction along $<_{\text {lex }}$ on $\left(l, k_{l}\right)$. The case $k_{l}>1$ is trivial since $\beta$ is then a multiple of o $\left(\boldsymbol{\beta}_{\uparrow_{l, k_{l}-1}}\right)$. Assume now that $k_{l}=1$, so that $\tau_{l, 1} \geq \tau$. Let $(u, v):=l^{\star}$, so $\left(n, m_{n}-1\right) \leq_{\text {lex }}(u, v)$ and $\tau_{l}^{\star} \geq \tau$. By Lemma $5.10(\mathrm{~b})$ of [5] we have

$$
\tilde{\tau}_{l, 1}=\kappa_{\tau_{l, 1}}^{\tilde{\tau}_{l}^{\star}}
$$

If $\left(n, m_{n}-1\right)=(u, v)$ we obtain $\tilde{\tau} \mid \kappa_{\tau_{l, 1}}^{\tilde{\tau}}$ since $\tau_{l, 1} \geq \tau$. By the i.h. we have $\tilde{\tau} \mid$ o $\left(\boldsymbol{\beta}_{\lceil u, v}\right)$, so $\tilde{\tau} \mid \tilde{\tau}_{u, v}$, and since $\tau_{l, 1} \geq \tau_{l}^{\star}$ we also have $\tilde{\tau} \mid \tilde{\tau}_{l, 1}$, which implies that $\tilde{\tau} \mid \beta$, cf. Definition 4.1.

$3 \Rightarrow \mathbf{2}$ : Assume there exists a maximal $j \in\{n+1, \ldots, l\}$ such that $\tau_{j, 1}<\tau$. Then we have $\tau_{j}^{\star} \leq \tau_{j, 1}<\tau$, so $(u, v):=j^{\star}<_{\text {lex }}\left(n, m_{n}-1\right)$. Let $\operatorname{ts}(\tilde{\tau})=:\left(\sigma_{1}, \ldots, \sigma_{s}\right)$ and recall Theorem 3.19 .

Case 1: $\tau_{j, 1} \notin \mathbb{E}^{>\tau_{j}^{\star}}$. Then it follows that $(j, 1)=\left(l, k_{l}\right)$ since $j$ was chosen maximally. In the case $\tau_{j}^{\star}=1$ we have $\tau_{j, 1}<\sigma_{1}$ and hence $\tilde{\tau}_{j, 1}=\kappa_{\tau_{j, 1}}<\tilde{\tau}$. Otherwise, i.e. $\tau_{j}^{\star}=\tau_{u, v}>1$, we obtain $\operatorname{ts}\left(\tilde{\tau}_{u, v}\right)=\operatorname{cs}\left(\boldsymbol{\alpha}_{\uparrow_{u, v}}\right)<_{\text {lex }} \operatorname{ts}(\tilde{\tau})$, so

$$
\tilde{\tau}_{j, 1}=\kappa_{\tau_{j, 1}}^{\mathrm{ts}\left(\tilde{\tau}_{u, v}\right)}<\tilde{\tau} .
$$

Case 2: $\tau_{j, 1} \in \mathbb{E}^{>\tau_{j}^{\star}}$. Then $\operatorname{ts}\left(\tilde{\tau}_{j, 1}\right)=\operatorname{cs}\left(\boldsymbol{\beta}_{\uparrow_{j, 1}}\right)=\operatorname{cs}\left(\boldsymbol{\beta}_{\uparrow_{u, v}}\right) \frown \tau_{j, 1}<_{\text {lex }} \operatorname{ts}(\tilde{\tau})$, hence $\tilde{\tau}_{j, 1}<\tilde{\tau}$. In the case $\tau_{l, k_{l}} \in \mathbb{E}^{>\tau_{l, k_{l}}^{\prime}}$ we see that $\operatorname{ts}\left(\tilde{\tau}_{j, 1}\right) \subseteq \operatorname{ts}\left(\tilde{\tau}_{l, k_{l}}\right)$, hence $\tilde{\tau}_{l, k_{l}}<\tilde{\tau}$. The other cases are easier, cf. Case 1 .

Applying the mappings tc, cf. Section 6 of [5], and o, which we verified in the present article to be elementary recursive, cf. Subsection [5.1, we are now able to formulate the arithmetical characterization of $\mathcal{C}_{2}$. 
Corollary 5.8 The structure $\mathcal{C}_{2}$ is characterized elementary recursively by

1. $\left(1^{\infty}, \leq\right)$ is the standard ordering of the classical notation system $1^{\infty}=\mathrm{T}^{1} \cap \Omega_{1}$, cf. [7]|,

2. $\alpha \leq_{1} \beta$ if and only if $\alpha \leq \beta \leq \operatorname{lh}(\alpha)$ where lh is given by equation 1 , and

3. $\alpha \leq_{2} \beta$ if and only if $\operatorname{tc}(\alpha) \subseteq \operatorname{tc}(\beta)$ and condition 2 of Lemma 5.7 holds.

Corollary 5.9 Let $M \subseteq_{\text {fin }} \mathrm{TC}$ be spanning (weakly spanning above some $\boldsymbol{\alpha} \in \mathrm{TC}$ ). Then $M$ is closed under lh .

Proof. This follows from Lemma 5.4] using Lemma 5.7] cf. Corollaries 5.6 and 7.13 of [5].

Recall Definition 5.13 of [5] which characterizes the standard linear ordering $\leq$ on $1^{\infty}$ by an ordering $\leq$ TC on the corresponding tracking chains. We can formulate a characterization of the relation $\leq_{1}$ (below $1^{\infty}$ ) in terms of the corresponding tracking chains as well. This follows from an inspection of the ordering $\leq_{\mathrm{TC}}$ in combination with the above statements. Let $\alpha, \beta<1^{\infty}$ with tracking chains tc $(\alpha)=\boldsymbol{\alpha}=\left(\boldsymbol{\alpha}_{1}, \ldots, \boldsymbol{\alpha}_{n}\right), \boldsymbol{\alpha}_{i}=\left(\alpha_{i, 1}, \ldots, \alpha_{i, m_{i}}\right)$, $1 \leq i \leq n$, and $\operatorname{tc}(\beta)=\boldsymbol{\beta}=\left(\boldsymbol{\beta}_{1}, \ldots, \boldsymbol{\beta}_{l}\right), \boldsymbol{\beta}_{i}=\left(\beta_{i, 1}, \ldots, \beta_{i, k_{i}}\right), 1 \leq i \leq l$. We have $\alpha \leq{ }_{1} \beta$ if and only if either $\boldsymbol{\alpha} \subseteq \boldsymbol{\beta}$ or there exists $(i, j) \in \operatorname{dom}(\boldsymbol{\alpha}) \cap \operatorname{dom}(\boldsymbol{\beta}), j<\min \left\{m_{i}, k_{i}\right\}$, such that $\boldsymbol{\alpha}_{\uparrow_{i, j}}=\boldsymbol{\beta}_{\uparrow_{i, j}}$ and $\alpha_{i, j+1}<\beta_{i, j+1}$, and we either have $\chi^{\tau_{i, j}}\left(\alpha_{i, j+1}\right)=0 \&(i, j+1)=\left(n, m_{n}\right)$ or $\chi^{\tau_{i, j}}\left(\alpha_{i, j+1}\right)=1 \& \alpha \leq{ }_{1} \mathrm{o}\left(\operatorname{me}\left(\boldsymbol{\alpha}_{\uparrow_{i, j+1}}\right)\right)<_{1} \beta$. Iterating this argument and recalling Lemma 5.5 of [5] we obtain the following

Proposition 5.10 Let $\alpha$ and $\beta$ with tracking chains $\boldsymbol{\alpha}$ and $\boldsymbol{\beta}$, respectively, as above. We have $\alpha \leq_{1} \beta$ if and only if either $\boldsymbol{\alpha} \subseteq \boldsymbol{\beta}$ or there exists the $<_{\text {lex }}$-increasing chain of index pairs $\left(i_{1}, j_{1}+1\right), \ldots,\left(i_{s}, j_{s}+1\right) \in \operatorname{dom}(\boldsymbol{\alpha})$ of maximal length $s \geq 1$ where $j_{r} \geq 1$ for $r=1, \ldots, s$, such that $\left(i_{1}, j_{1}+1\right) \in \operatorname{dom}(\boldsymbol{\beta}), \boldsymbol{\alpha}_{\uparrow_{i_{1}, j_{1}}}=\boldsymbol{\beta}_{\uparrow_{i_{1}, j_{1}}}$, $\alpha_{i_{1}, j_{1}+1}<\beta_{i_{1}, j_{1}+1}$

$$
\boldsymbol{\alpha}_{\uparrow_{i_{s}, j_{s}+1}} \subseteq \boldsymbol{\alpha} \subseteq \operatorname{me}\left(\boldsymbol{\alpha}_{\uparrow_{i_{s}, j_{s}+1}}\right)
$$

and $\chi^{\tau_{i_{r}, j_{r}}}\left(\alpha_{i_{r}, j_{r}+1}\right)=1$ at least whenever $\left(i_{r}, j_{r}+1\right) \neq\left(n, m_{n}\right)$. Setting $\alpha_{r}:=\mathrm{o}\left(\boldsymbol{\alpha}_{\uparrow_{i_{r}, j_{r}+1}}\right)$ for $r=1, \ldots, s$ as well as $\alpha_{r}^{+}:=\mathrm{O}\left(\operatorname{me}\left(\boldsymbol{\alpha}_{\uparrow_{i_{r}, j_{r}+1}}\right)\right)$ for $r$ such that $\chi^{\tau_{i_{r}, j_{r}}}\left(\alpha_{i_{r}, j_{r}+1}\right)=1$ and $\alpha_{s}^{+}:=\alpha$ if $\chi^{\tau_{i_{s}, j_{s}}}\left(\alpha_{i_{s}, j_{s}+1}\right)=0$ we have

$$
\alpha_{1}<_{2} \ldots<_{2} \alpha_{s} \leq_{2} \alpha \leq_{1} \alpha_{s}^{+}<_{1} \ldots<_{1} \alpha_{1}^{+}<_{1} \mathrm{o}\left(\boldsymbol{\beta}_{\uparrow_{i_{1}, j_{1}+1}}\right) \leq_{1} \beta
$$

For $\beta=\operatorname{lh}(\alpha)$ the cases $\boldsymbol{\alpha} \subseteq \boldsymbol{\beta}$ and $s=1$ with $\left(i_{1}, j_{1}+1\right)=\left(n, m_{n}\right)$ correspond to the situation $\mathrm{gbo}(\boldsymbol{\alpha})=\left(n, m_{n}\right)$, while otherwise we have $\operatorname{gbo}(\boldsymbol{\alpha})=\left(i_{1}, j_{1}+1\right)$.

Remark. Note that the above index pairs characterize the relevant sub-maximal $\nu$-indices in the initial chains of $\boldsymbol{\alpha}$ with respect to $\boldsymbol{\beta}$ and leave the intermediate steps of maximal (me-) extension along the iteration. Using Lemma 5.5 of [5] we observe that the sequence $\tau_{i_{1}, j_{1}}, \ldots, \tau_{i_{s}, j_{s}}$ of bases in the above proposition satisfies

$$
\tau_{i_{1}, j_{1}}<\ldots<\tau_{i_{s}, j_{s}} \quad \text { and } \quad \tau_{i_{s}, j_{s}}<\tau_{i, 1} \quad \text { for every } i \in\left(i_{s}, n\right] \text {, }
$$

so that in the case where $\alpha<_{1} \beta$ and $\boldsymbol{\alpha} \nsubseteq \boldsymbol{\beta}$ we have $\alpha<_{1} \operatorname{gs}(\alpha) \leq_{1} \beta$ with $\tau_{i_{s}, j_{s}} \mid \rho_{n} \dot{-}$.

Lemma 5.11 The relation $\subseteq$ of initial chain on $\mathrm{TC}$ respects the ordering $\leq_{\mathrm{TC}}$ and hence also the characterization of $\leq_{1}$ on $\mathrm{TC}$.

Proof. Suppose tracking chains $\boldsymbol{\alpha}, \boldsymbol{\beta}, \boldsymbol{\gamma} \in \mathrm{TC}$ satisfy $\boldsymbol{\alpha} \leq_{\mathrm{TC}} \boldsymbol{\beta} \leq_{\mathrm{TC}} \boldsymbol{\gamma}$ and $\boldsymbol{\alpha} \subseteq \boldsymbol{\gamma}$. The case where any two chains are equal is trivial. We may therefore assume that $\mathrm{o}(\boldsymbol{\alpha})<\mathrm{o}(\boldsymbol{\beta})<\mathrm{o}(\boldsymbol{\gamma})$ as $\left(\mathrm{TC}, \leq_{\mathrm{TC}}\right)$ and $\left(1^{\infty},<\right)$ are order-isomorphic. Since $\boldsymbol{\alpha} \subseteq \gamma$ we have o $(\boldsymbol{\alpha})<_{1} \mathrm{o}(\boldsymbol{\gamma})$, hence $\mathrm{o}(\boldsymbol{\alpha})<_{1} \mathrm{o}(\boldsymbol{\beta})$ as $\leq{ }_{1}$ respects $\leq$. Assume toward contradiction that $\boldsymbol{\alpha} \nsubseteq \boldsymbol{\beta}$, whence by Proposition 5.10 there exists $(i, j+1) \in \operatorname{dom}(\boldsymbol{\alpha}) \cap \operatorname{dom}(\boldsymbol{\beta}) \operatorname{such} \operatorname{that}$ $\boldsymbol{\alpha}_{\uparrow_{i, j}}=\boldsymbol{\beta}_{\uparrow_{i, j}}$ and $\alpha_{i, j+1}<\beta_{i, j+1}$. But then for any $\boldsymbol{\delta} \in$ TC such that $\boldsymbol{\alpha} \subseteq \boldsymbol{\delta}$ we have

$$
\mathrm{o}(\boldsymbol{\delta})<\mathrm{o}\left(\boldsymbol{\alpha}^{+}\right) \leq \mathrm{o}(\boldsymbol{\beta})<\mathrm{o}(\boldsymbol{\gamma})
$$

where the tracking chain $\boldsymbol{\alpha}^{+}:=\boldsymbol{\alpha}_{\uparrow_{i, j+1}}\left[\alpha_{i, j+1}+1\right]$ is the result of changing the terminal index $\alpha_{i, j+1}$ of $\boldsymbol{\alpha}_{\uparrow_{i, j+1}}$ to $\alpha_{i, j+1}+1$.

We may now return to the issue of closure under lh, which has a convenient sufficient condition on the basis of the following 
Definition 5.12 A tracking chain $\boldsymbol{\alpha} \in \mathrm{TC}$ is called convex if and only if every $\nu$-index in $\boldsymbol{\alpha}$ is maximal, i.e. given by the corresponding $\mu$-operator.

Corollary 5.13 Let $\boldsymbol{\alpha} \in \mathrm{TC}$ be convex and $M \subseteq_{\text {fin }}$ TC be weakly spanning above $\boldsymbol{\alpha}$. Then $M$ is closed under lh.

Proof. This is a consequence of Proposition [5.10, Corollary [5.5, Lemma [5.4, and equation 7 ,

While it is easy to observe that in $\mathcal{R}_{2}$ the relation $\leq_{1}$ is a forest that respects $\leq$ and the relation $\leq_{2}$ is a forest contained in $\leq_{1}$ which respects $\leq_{1}$, we can now conclude that this also holds for the arithmetical formulations of $\leq_{1}$ and $\leq_{2}$ in $\mathcal{C}_{2}$, without referring to the results in Section 7 of [5].

Corollary 5.14 Consider the arithmetical characterizations of $\leq_{1}$ and $\leq_{2}$ on $1^{\infty}$. The relation $\leq_{2}$ respects $\leq_{1}$, i.e. whenever $\alpha \leq_{1} \beta \leq_{1} \gamma<1^{\infty}$ and $\alpha \leq_{2} \gamma$, then $\alpha \leq_{2} \beta$.

Proof. In the case $\boldsymbol{\beta} \subseteq \gamma$ this directly follows from Lemma 5.7, while otherwise we additionally employ Proposition 5.10 and property 9 .

\section{Conclusion}

In the present article, the arithmetical characterization of the structure $\mathcal{C}_{2}$, which was established in Theorem 7.9 and Corollary 7.13 of [5], has been analyzed and shown to be elementary recursive. We have seen that finite isomorphism types of $\mathcal{C}_{2}$ (in its arithmetical formulation) are contained in the class of "respecting forests", cf. [2], over the language $\left(\leq_{1}, \leq_{1}, \leq_{2}\right)$. In a subsequent article [11] we will establish the converse by providing an effective assignment of isominimal realizations in $\mathcal{C}_{2}$ to arbitrary respecting forests. We will provide an algorithm to find pattern notations for the ordinals in $\mathcal{C}_{2}$, and will conclude that the union of isominimal realizations of respecting forests is indeed the core of $\mathcal{R}_{2}$, i.e. the structure $\mathcal{C}_{2}$ in its semantical formulation based on $\Sigma_{i^{-}}$ elementary substructures, $i=1,2$. As a corollary we will see that the well-quasi orderedness of respecting forests with respect to coverings, which was shown by Carlson in [3], implies (in a weak theory) transfinite induction up to the proof-theoretic ordinal $1^{\infty}$ of $\mathrm{KP} \ell_{0}$.

We expect that the approaches taken here and in our treatment of the structure $\mathcal{R}_{1}^{+}$, see [8] and [9], will naturally extend to an analysis of the structure $\mathcal{R}_{2}^{+}$and possibly to structures of patterns of higher order. A subject of ongoing work is to verify that the core of $\mathcal{R}_{2}^{+}$matches the proof-theoretic strength of a limit of KPImodels.

\section{Acknowledgements}

I would like to express my gratitude to Professor Ulf Skoglund for encouragement and support of my research. I would like to thank Dr. Steven D. Aird for editing the manuscript.

\section{References}

[1] T. J. Carlson: Ordinal Arithmetic and $\Sigma_{1}$-Elementarity. Archive for Mathematical Logic 38 (1999) $449-460$.

[2] T. J. Carlson: Elementary Patterns of Resemblance. Annals of Pure and Applied Logic 108 (2001) 19-77.

[3] T. J. Carlson: Generalizing Kruskal's theorem to pairs of cohabitating trees. Archive for Mathematical Logic 55 (2016) $37-48$.

[4] T. J. Carlson and G. Wilken: Normal Forms for Elementary Patterns. The Journal of Symbolic Logic 77 (2012) 174-194.

[5] T. J. Carlson and G. Wilken: Tracking Chains of $\Sigma_{2}$-Elementarity. Annals of Pure and Applied Logic 163 (2012) 23-67.

[6] W. Pohlers: Proof Theory. The First Step into Impredicativity. Springer, Berlin 2009.

[7] G. Wilken: Ordinal Arithmetic based on Skolem Hulling. Annals of Pure and Applied Logic 145 (2007) $130-161$.

[8] G. Wilken: $\Sigma_{1}$-Elementarity and Skolem Hull Operators. Annals of Pure and Applied Logic 145 (2007) 162-175. 
[9] G. Wilken: Assignment of Ordinals to Elementary Patterns of Resemblance. The Journal of Symbolic Logic 72 (2007) 704-720.

[10] G. Wilken: Tracking chains revisited. S.-D. Friedman, D. Raghavan, Y. Yang (eds.): Sets and Computations, Lecture Notes Series Vol. 33, Institute for Mathematical Sciences, National University of Singapore, World Scientific Publishing Company (2017) 183-220.

[11] G. Wilken: Pure patterns of order 2. Annals of Pure and Applied Logic (2017), in press. DOI: 10.1016/j.apal.2017.09.001 